Bulletin of the Section of Logic

Volume 49/1 (2020), pp. 13-51

http://dx.doi.org/10.18778/0138-0680.2020.02

Aldo V. Figallo, Inés Pascual, Gustavo Pelaitay

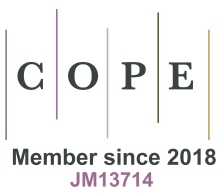

\title{
A TOPOLOGICAL APPROACH TO TENSE $\mathrm{LM}_{n \times m}$-ALGEBRAS
}

\begin{abstract}
In 2015, tense $n \times m$-valued Eukasiewicz-Moisil algebras (or tense $L M_{n \times m^{-}}$ algebras) were introduced by A. V. Figallo and G. Pelaitay as an generalization of tense $n$-valued Łukasiewicz-Moisil algebras. In this paper we continue the study of tense $L M_{n \times m}$-algebras. More precisely, we determine a Priestley-style duality for these algebras. This duality enables us not only to describe the tense $L M_{n \times m}$-congruences on a tense $L M_{n \times m}$-algebra, but also to characterize the simple and subdirectly irreducible tense $L M_{n \times m}$-algebras.
\end{abstract}

Keywords: Tense $L M_{n \times m}$-algebras, Priestley-style topological duality, Priestley spaces, tense De Morgan algebras.

\section{Introduction}

In 1975, Suchoń ([36]) defined matrix Łukasiewicz algebras so generalizing $n$-valued Łukasiewicz algebras without negation ([29]). In 2000, A. V. Figallo and C. Sanza ([23]) introduced $n \times m$-valued Łukasiewicz algebras with negation which are both a particular case of matrix Łukasiewicz algebras and a generalization of $n$-valued Eukasiewicz-Moisil algebras ([1]). It is worth noting that unlike what happens in $n$-valued Lukasiewicz-Moisil algebras, generally the De Morgan reducts of $n \times m$-valued Łukasiewicz algebras with negation are not Kleene algebras. Furthermore, in [34] an important example which legitimated the study of this new class of algebras is provided. Following the terminology established in [1], these 
algebras were called $n \times m$-valued Lukasiewicz-Moisil algebras (or $\mathrm{LM}_{n \times m^{-}}$ algebras for short). $L M_{n \times m}$-algebras were studied in $[24,25,15,34]$ and [35].

Propositional logics usually do not incorporate the dimension of time; consequently, in order to obtain a tense logic, a propositional logic is enriched by the addition of new unary operators (or connectives) which are usually denoted by $G, H, F$ and $P$. We can define $F$ and $P$ by means of $G$ and $H$ as follows: $F(x)=\neg G(\neg x)$ and $P(x)=\neg H(\neg x)$, where $\neg x$ denotes negation of the proposition $x$. Tense algebras (or tense Boolean algebras) are algebraic structures corresponding to the propositional tense logic (see $[4,19]$ ). An algebra $\langle A, \vee, \wedge, \neg, G, H, 0,1\rangle$ is a tense algebra if $\langle A, \vee, \wedge, \neg, 0,1\rangle$ is a Boolean algebra and $G, H$ are unary operators on $A$ which satisfy the following axioms for all $x, y \in A$ :

$$
\begin{gathered}
G(1)=1, H(1)=1, \\
G(x \wedge y)=G(x) \wedge G(y), H(x \wedge y)=H(x) \wedge H(y), \\
x \leq G P(x), x \leq H F(x),
\end{gathered}
$$

where $P(x)=\neg H(\neg x)$ and $F(x)=\neg G(\neg x)$.

Taking into account that tense algebras constitute the algebraic basis for the bivalent tense logic, D. Diaconescu and G. Georgescu introduced in [12] the tense $M V$-algebras and the tense Lukasiewicz-Moisil algebras (or tense $n$-valued Lukasiewicz-Moisil algebras) as algebraic structures for some many-valued tense logics. In recent years, these two classes of algebras have become very interesting for several authors (see $[2,6,8,9$, $15,7,17,18])$. In particular, in $[8,9]$, Chiriţă, introduced tense $\theta$-valued Lukasiewicz-Moisil algebras and proved an important representation theorem which made it possible to show the completeness of the tense $\theta$-valued Moisil logic (see [8]). In [12], the authors formulated an open problem about representation of tense $M V$-algebras, this problem was solved in [21,3] for semisimple tense $M V$-algebras. Also, in [2], tense basic algebras which are an interesting generalization of tense $M V$-algebras, were studied.

The main purpose of this paper is to give a topological duality for tense $n \times m$-valued Łukasiewicz-Moisil algebras. In order to achieve this we will extend the topological duality given in [27], for $n \times m$-valued LukasiewiczMoisil algebras. In [35] another duality for $n \times m$-valued Lukasiewicz-Moisil algebras was developed, starting from De Morgan spaces and adding a family of continuous functions. 
The paper is organized as follows: In Section 2, we briefly summarize the main definitions and results needed throughout this article. In Section 3, we developed a topological duality for tense $n \times m$-valued Łukasiewicz-Moisil algebras, extending the one obtained in [27] for $n \times m$ valued Łukasiewicz-Moisil algebras. In Section 4, the results of Section 3 are applied. Firstly, we characterize congruences on tense $n \times m$-valued Łukasiewicz-Moisil algebras by certain closed and increasing subsets of the space associated with them. This enables us to describe the subdirectly irreducible tense $n \times m$-valued Łukasiewicz-Moisil algebras and the simple tense $n \times m$-valued Łukasiewicz-Moisil algebras.

\section{Preliminaries}

\subsection{Tense De Morgan algebras}

In [16] A. V. Figallo and G. Pelaitay introduced the variety of algebras, which they call tense De Morgan algebras, and they also developed a representation theory for this class of algebras.

First, recall that an algebra $\langle A, \vee, \wedge, \sim, 0,1\rangle$ is a De Morgan algebra if $\langle A, \vee, \wedge, 0,1\rangle$ is a bounded distributive lattice and $\sim$ is a unary operation on $A$ satisfying the following identities for all $x, y \in A$ :

1. $\sim(x \vee y)=\sim x \wedge \sim y$,

2. $\sim \sim x=x$,

3. $\sim 0=1$.

In what follows a De Morgan algebra $\langle A, \vee, \wedge, \sim, 0,1\rangle$ will be denoted briefly by $(A, \sim)$.

Definition 1. An algebra $(A, \sim, G, H)$ is a tense De Morgan algebra if $(A, \sim)$ is a De Morgan algebra and $G$ and $H$ are two unary operations on $A$ such that for any $x, y \in A$ :

1. $G(1)=1$ and $H(1)=1$,

2. $G(x \wedge y)=G(x) \wedge G(y)$ and $H(x \wedge y)=H(x) \wedge H(y)$,

3. $x \leq G P(x)$ and $x \leq H F(x)$, where $F(x)=\sim G(\sim x)$ and $P(x)=\sim$ $H(\sim x)$

4. $G(x \vee y) \leq G(x) \vee F(y)$ and $H(x \vee y) \leq H(x) \vee P(y)$.

In [16] a duality for tense De Morgan algebras is described taking into account the results established by W. Cornish and P. Fowler in [11]. To this 
purpose, the topological category tmPS of $t m P$-spaces and $t m P$-functions was considered, which we indicate below:

Definition 2. A tense De Morgan space (or tmP-space) is a system $\left(X, g, R, R^{-1}\right)$, where

(i) $(X, g)$ is an $m P$-space $([11])$. More precisely,

(mP1) $X$ is a Priestley space (or $P$-space),

(mP2) $g: X \longrightarrow X$ is an involutive homeomorphism and an antiisomorphism,

(ii) $R$ is a binary relation on $X$ and $R^{-1}$ is the converse of $R$ such that:

(tS1) For each $U \in D(X)$ it holds that $G_{R}(U), H_{R^{-1}}(U) \in D(X)$, where $G_{R}$ and $H_{R^{-1}}$ are two operators on $\mathcal{P}(X)$ defined for any $U \subseteq X$ as follows:

$$
\begin{gathered}
G_{R}(U)=\{x \in X \mid R(x) \subseteq U\}, \\
H_{R^{-1}}(U)=\left\{x \in X \mid R^{-1}(x) \subseteq U\right\},
\end{gathered}
$$

and $D(X)$ is the set of all increasing and clopen subsets of $X$,

(tS2) $(x, y) \in R$ implies $(g(x), g(y)) \in R$ for any $x, y \in X$,

(tS3) for each $x \in X, R(x)$ is a closed set in $X$,

(tS4) for each $x \in X, R(x)=\downarrow R(x) \cap \uparrow R(x)$, where $\downarrow Y(\uparrow Y)$ denotes the set of all $x \in X$ such that $x \leq y(y \leq x)$ for some $y \in Y \subseteq X$.

Definition 3. A tmP-function from a $t m P$-space $\left(X_{1}, g_{1}, R_{1}, R_{1}^{-1}\right)$ into another one, $\left(X_{2}, g_{2}, R_{2}, R_{2}^{-1}\right)$, is a continuous and increasing function (P-function) $f: X_{1} \longrightarrow X_{2}$, which satisfies the following conditions:

(mf) $f \circ g_{1}=g_{2} \circ f(m P$-function [11]),

(tf1) $(x, y) \in R_{1}$ implies $(f(x), f(y)) \in R_{2}$ for any $x, y \in X_{1}$,

(tf2) if $(f(x), y) \in R_{2}$, then there is an element $z \in X_{1}$ such that $(x, z) \in$ $R_{1}$ and $f(z) \leq y$

(tf3) if $(y, f(x)) \in R_{2}$, then there is an element $z \in X_{1}$ such that $(z, x) \in$ $R_{1}$ and $f(z) \leq y$.

Next, A. V. Figallo and G. Pelaitay (see [16, Section 5]) showed that the category tmPS is dually equivalent to the category TDMA of tense De Morgan algebras and tense De Morgan homomorphisms. The following results are used to show the dual equivalence: 
- Let $\left(X, g, R, R^{-1}\right)$ be a tmP-space. Then, $\left(D(X), \sim_{g}, G_{R}, H_{R^{-1}}\right)$ is a tense De Morgan algebra, where for all $U \in D(X), \sim_{g} U$ is defined by

$$
\sim_{g} U=X \backslash g(U)
$$

and $G_{R}(U)$ and $H_{R^{-1}}(U)$ are defined as in (2.1) and (2.2), respectively.

- Let $(A, \sim, G, H)$ be a tense De Morgan algebra and $X(A)$ be the Priestley space associated with $A$, i.e. $X(A)$ is the set of all prime filters of $A$, ordered by inclusion and with the topology having as a sub-basis the following subsets of $X(A)$ :

$$
\sigma_{A}(a)=\{S \in X(A): a \in S\} \text { for each } a \in A,
$$

and

$$
X(A) \backslash \sigma_{A}(a) \text { for each } a \in A .
$$

Then, $\left(X(A), g_{A}, R_{G}^{A}, R_{H}^{A}\right)$ is a $t m P$-space, where $g_{A}(S)$ is defined by

$$
g_{A}(S)=\{x \in A: \sim x \notin S\}, \text { for all } S \in X(A),
$$

and the relations $R_{G}^{A}$ and $R_{H}^{A}$ are defined for all $S, T \in X(A)$ as follows:

$$
\begin{aligned}
& (S, T) \in R_{G}^{A} \Longleftrightarrow G^{-1}(S) \subseteq T \subseteq F^{-1}(S), \\
& (S, T) \in R_{H}^{A} \Longleftrightarrow H^{-1}(S) \subseteq T \subseteq P^{-1}(S) .
\end{aligned}
$$

- Let $(A, \sim, G, H)$ be a tense De Morgan algebra; then, the function $\sigma_{A}: A \longrightarrow D(X(A))$ is a tense De Morgan isomorphism, where $\sigma_{A}$ is defined as in (2.4).

- Let $\left(X, g, R, R^{-1}\right)$ be a $t m P$-space; then, $\varepsilon_{X}: X \longrightarrow X(D(X))$ is an isomorphism of $t m P$-spaces, where $\varepsilon_{X}$ is defined by

$$
\varepsilon_{X}(x)=\{U \in D(X): x \in U\}, \text { for all } x \in X .
$$


- Let $h:\left(A_{1}, \sim_{1}, G_{1}, H_{1}\right) \longrightarrow\left(A_{2}, \sim_{2}, G_{2}, H_{2}\right)$ be a tense De Morgan morphism. Then, the map $\Phi(h): X\left(A_{2}\right) \longrightarrow X\left(A_{1}\right)$ is a morphism of $t m P$-spaces, where

$$
\Phi(h)(S)=h^{-1}(S), \text { for all } S \in X\left(A_{2}\right) .
$$

- Let $f:\left(X_{1}, g_{1}, R_{1}, R_{1}^{-1}\right) \longrightarrow\left(X_{2}, g_{2}, R_{2}, R_{2}^{-1}\right)$ be a morphism of tmP-spaces. Then, $\Psi(f): D\left(X_{2}\right) \longrightarrow D\left(X_{1}\right)$ is a tense De Morgan morphism, where

$$
\Psi(f)(U)=f^{-1}(U), \text { for all } U \in D\left(X_{2}\right) .
$$

In [16], the duality described above was used to characterize the congruence lattice $\operatorname{Con}_{t M}(A)$ of a tense De Morgan algebra $(A, \sim, G, H)$. First the following notion was introduced:

Definition 4. Let $\left(X, \leq, g, R, R^{-1}\right)$ be a $t m P$-space. An involutive (i.e. $Y=g(Y)$ [11] ) closed subset $Y$ of $X$ is a $t m P$-subset if it satisfies the following conditions for $u, v \in X$ :

(ts1) if $(v, u) \in R$ and $u \in Y$, then there exists, $w \in Y$ such that $(w, u) \in R$ and $w \leq v$.

(ts2) if $(u, v) \in R$ and $u \in Y$, then there exists, $z \in Y$ such that $(u, z) \in R$ and $z \leq v$.

The lattice of all $t m P$-subsets of the $t m P$-space associated with a tense De Morgan algebra was taken into account to characterize the congruence lattice of this algebra as it is indicated in the following theorem:

TheOrem 1. ([16, Theorem 6.4]) Let $(A, \sim, G, H)$ be a tense De Morgan algebra and $\left(X(A), \subseteq, g_{A}, R_{G}^{A}, R_{H}^{A}\right)$ be the tmP-space associated with $A$. Then, the lattice $\mathcal{C}_{T}(X(A))$ of all tmP-subsets of $X(A)$ is anti-isomorphic to the lattice $\operatorname{Con}_{t M}(A)$ of the tense De Morgan congruences on $A$, and the anti-isomorphism is the function $\Theta_{T}$ defined by the prescription:

$\Theta_{T}(Y)=\left\{(a, b) \in A \times A: \sigma_{A}(a) \cap Y=\sigma_{A}(b) \cap Y\right\}$, for all $Y \in \mathcal{C}_{T}(X(A))$.

\section{2. $n \times m$-valued Łukasiewicz-Moisil algebras}

In the sequel $n$ and $m$ are positive integer numbers and we use the notation $[n]:=\{1, \ldots, n-1\}$ and so the cartesian product $\{1, \ldots, n-1\} \times$ $\{1, \ldots, m-1\}$ is denoted by $[n] \times[m]$. 
Definition 5. ([34, Definition 3.1.]) Let $n \geq 2$ and $m \geq 2$. An $n \times$ $m$-valued Lukasiewicz-Moisil algebra (or $\mathrm{LM}_{n \times m}$-algebra) is an algebra $\left\langle A, \wedge, \vee, \sim,\left\{\sigma_{i j}\right\}_{(i, j) \in[n] \times[m]}, 0,1\right\rangle$, such that:

(a) the reduct $\langle A, \wedge, \vee, \sim, 0,1\rangle$ is a De Morgan algebra,

(b) $\left\{\sigma_{i j}\right\}_{(i, j) \in[n] \times[m]}$ is a family of unary operations on $A$ which fulfills the following conditions for any $x, y \in A$ and any $(i, j),(r, s) \in[n] \times[m]$ :

(C1) $\sigma_{i j}(x \vee y)=\sigma_{i j} x \vee \sigma_{i j} y$,

(C2) $\sigma_{i j} x \leq \sigma_{(i+1) j} x$,

(C3) $\sigma_{i j} x \leq \sigma_{i(j+1)} x$,

(C4) $\sigma_{i j} \sigma_{r s} x=\sigma_{r s} x$,

(C5) $\sigma_{i j} x=\sigma_{i j} y$ for all $(i, j) \in[n] \times[m]$ imply $x=y$,

(C6) $\sigma_{i j} x \vee \sim \sigma_{i j} x=1$,

(C7) $\sigma_{i j}(\sim x)=\sim \sigma_{(n-i)(m-j)} x$.

In what follows and where no confusion might arise, we denote these algebras by $A$ or $\left(A, \sim,\left\{\sigma_{i j}\right\}_{(i, j) \in[n] \times[m]}\right)$, in the case we need to specify unary operators.

In Lemma 1 we summarize the most important properties of these algebras necessary in what follows.

Lemma 1. ([34, Lemma 3.1.]) Let $\left(A, \sim,\left\{\sigma_{i j}\right\}_{(i, j) \in[n] \times[m]}\right)$ be an $L M_{n \times m^{-}}$ algebra. Then, the following properties are satisfied for all $x, y \in A$ and for all $(i, j) \in[n] \times[m]$ :

(C8) $\sigma_{i j}(x \wedge y)=\sigma_{i j} x \wedge \sigma_{i j} y$

(C9) $\sigma_{i j} x \wedge \sim \sigma_{i j} x=0$,

(C10) $x \leq y$ if and only if $\sigma_{i j} x \leq \sigma_{i j} y$ for all $(i, j) \in[n] \times[m]$,

(C11) $x \leq \sigma_{(n-1)(m-1)} x$,

(C12) $\sigma_{i j} 0=0, \sigma_{i j} 1=1$,

(C13) $\sigma_{11} x \leq x$,

(C14) $\sim x \vee \sigma_{(n-1)(m-1)} x=1$,

(C15) $x \vee \sim \sigma_{11} x=1$.

Definition 6. ([28, Definition 2.1.]) Let $\left(A, \sim,\left\{\sigma_{i j}\right\}_{(i, j) \in[n] \times[m]}\right)$ and $\left(A^{\prime}, \sim^{\prime},\left\{\sigma_{i j}^{\prime}\right\}_{(i, j) \in[n] \times[m]}\right)$ be two $L M_{n \times m}$-algebras. A function $h: A \longrightarrow$ $A^{\prime}$ is an $L M_{n \times m}$-homomorphism if it satisfies the following conditions for all $x, y \in A$ and for all $(i, j) \in[n] \times[m]$ : 
(a) $h$ is a lattice homomorphism,

(b) $h(\sim x)=\sim^{\prime} h(x)$,

(c) $h\left(\sigma_{i j} x\right)=\sigma_{i j}^{\prime} h(x)$.

Lemma 2. ([28, Remark 2.2.]) Let $\left(A, \sim,\left\{\sigma_{i j}\right\}_{(i, j) \in[n] \times[m]}\right)$ and $\left(A^{\prime}, \sim^{\prime}\right.$, $\left.\left\{\sigma_{i j}^{\prime}\right\}_{(i, j) \in[n] \times[m]}\right)$ be two $L M_{n \times m}$-algebras and $h: A \longrightarrow A^{\prime}$ be a lattice homomorphism. Then the following conditions are equivalent:

(a) $h$ is an $L M_{n \times m}$-homomorphism,

(b) $h\left(\sigma_{i j} x\right)=\sigma_{i j}^{\prime} h(x)$ for all $x \in A$ and for all $(i, j) \in[n] \times[m]$.

The results announced here for $L M_{n \times m}$-algebras are used throughout the paper.

(LM1) $\sigma_{i j}(A)=B(A)$ for all $(i, j) \in[n] \times[m]$, where $B(A)$ is the set of all complemented elements of $A$ ([33, Proposition 2.5]).

(LM2) Every $L M_{n \times 2}$-algebra is isomorphic to an $n$-valued LukasiewiczMoisil algebra. It is worth noting that $L M_{n \times m}$-algebras constitute a non-trivial generalization of the latter (see [34, Remark 2.1]).

(LM3) The class of $L M_{n \times m}$-algebras is a variety and two equational bases for it can be found in [33, Theorem 2.7] and [34, Theorem 4.6].

(LM4) Let $X$ be a non-empty set and let $A^{X}$ be the set of all functions from $X$ into $A$. Then $A^{X}$ is an $L M_{n \times m}$-algebra, where the operations are defined componentwise.

(LM5) Let $B(A) \uparrow^{[n] \times[m]}=\{f:[n] \times[m] \longrightarrow B(A)$ such that for arbitrary $i, j$, if $r \leq s$, then $f(r, j) \leq f(s, j)$ and $f(i, r) \leq f(i, s)\}$. Then $\left\langle B(A) \uparrow^{[n] \times[m]}, \wedge, \vee, \sim,\left\{\sigma_{i j}\right\}_{(i, j) \in[n] \times[m]}, 0,1\right\rangle$ is an $L M_{n \times m}$-algebra, where for all $f \in B(A) \uparrow[n] \times[m]$ and $(i, j) \in[n] \times[m]$ the operations $\sim$ and $\sigma_{i j}$ are defined as follows:

$$
(\sim f)(i, j)=\neg f(n-i, m-j),
$$

where $\neg x$ is the Boolean complement of $x$,

$$
\left(\sigma_{i j} f\right)(r, s)=f(i, j) \text { for all }(r, s) \in[n] \times[m],
$$

and the remaining operations are defined componentwise ([34, Proposition 3.2]). It is worth noting that this result can be generalized by replacing $B(A)$ by any Boolean algebra $B$. Furthermore, if $B$ is a complete Boolean algebra, it is simple to check that $B \uparrow^{[n] \times[m]}$ is also a complete $L M_{n \times m}$-algebra. 
(LM6) Every $L M_{n \times m}$-algebra $\left(A, \sim,\left\{\sigma_{i j}\right\}_{(i, j) \in[n] \times[m]}\right)$ can be embedded into the $L M_{n \times m}$-algebra $B(A) \uparrow[n] \times[m]$ ([34, Theorem 3.1]). Besides, $A$ is isomorphic to $B(A) \uparrow[n] \times[m]$ if and only if $A$ is centred ([34, Corollary 3.1]), where $A$ is centred if for each $(i, j) \in[n] \times[m]$ there exists $c_{i j} \in A$ such that

$$
\sigma_{r s} c_{i j}= \begin{cases}0 & \text { if } i>r \text { or } j>s \\ 1 & \text { if } i \leq r \text { and } j \leq s .\end{cases}
$$

(LM7) Let $\mathbf{2} \uparrow[\mathbf{n}] \times[\mathbf{m}]$ be the set of all increasing functions from $[n] \times[m]$ to the Boolean algebra $\mathbf{2}$ with two elements. Then every simple $L M_{n \times m^{-}}$ algebra is a subalgebra of $\left\langle\mathbf{2} \uparrow[\mathbf{n}] \times[\mathbf{m}], \wedge, \vee, \sim,\left\{\sigma_{i j}\right\}_{(i, j) \in[n] \times[m]}, \mathbf{0}, \mathbf{1}\right\rangle$, where the operations of this $L M_{n \times m}$-algebra are defined as in statement (LM5) and $\mathbf{0 ,} \mathbf{1} \in \mathbf{2} \uparrow[\mathbf{n}] \times[\mathbf{m}]$ are the functions $\mathbf{0 , 1}:[n] \times$ $[m] \longrightarrow \mathbf{2}$, defined for all $(i, j) \in[n] \times[m]$ by $\mathbf{0}((i, j))=0$ and $\mathbf{1}((i, j))=1$, respectively (see [34, Theorem 5.5]).

(LM8) Let $A$ be an $L M_{n \times m}$-algebra. Then, the following conditions are equivalent:

(a) $A$ is a subdirectly irreducible $L M_{n \times m}$-algebra,

(b) $B(A)=\{0,1\}$, where $B(A)=\left\{\sigma_{i j} a: a \in A,(i, j) \in[n] \times[m]\right\}$.

In [27], A. V. Figallo, I. Pascual and G. Pelaitay determined a topological duality for $L M_{n \times m}$-algebras. To this aim, these authors considered the topological category $\mathbf{L M}_{\mathbf{n} \times \mathbf{m}} \mathbf{P}$ of $L M_{n \times m}$-spaces and $L M_{n \times m}$-functions. Specifically:

Definition 7. A system $\left(X, g,\left\{f_{i j}\right\}_{(i, j) \in[n] \times[m]}\right)$ is an $n \times m$-valued Eukasiewicz-Moisil space (or shortly $L M_{n \times m^{-}}$-space) if the following properties are fulfilled for all $x, y \in X$ and $(i, j),(r, s) \in[n] \times[m]$ :

(LP1) $(X, g)$ is an $m$-space,

(LP2) $f_{i j}: X \longrightarrow X$ is a continuous function,

(LP3) $f_{i j}(x) \leq f_{(i+1) j}(x)$,

(LP4) $f_{i j}(x) \leq f_{i(j+1)}(x)$,

(LP5) $x \leq y$ implies $f_{i j}(x)=f_{i j}(y)$ for all $(i, j) \in[n] \times[m]$,

(LP6) $f_{i j} \circ f_{r s}=f_{i j}$,

(LP7) $f_{i j} \circ g=f_{i j}$, 
(LP8) $g \circ f_{i j}=f_{(n-i)(m-j)}$,

(LP9) $\bigcup_{(i, j) \in[n] \times[m]} f_{i j}(X)=X$.

Remark 1. The axiom (LP5) is omitted in the Sanza's definition of $L M_{n \times m^{-}}$ space ( see [35, Definition 2.1]). This axiom plays a fundamental role in the characterization of $L M_{n \times m}$-spaces and consequently in the characterization of congruences on $L M_{n \times m}$-algebras as we prove next.

Definition 8. If $\left(X, g,\left\{f_{i j}\right\}_{(i, j) \in[n] \times[m]}\right)$ and $\left(X^{\prime}, g^{\prime},\left\{f_{i j}^{\prime}\right\}_{(i, j) \in[n] \times[m]}\right)$ are two $L M_{n \times m}$-spaces, then an $L M_{n \times m}$-function $f$ from $X$ to $X^{\prime}$ is a continuous and increasing function ( $P$-function), which satisfies the following conditions:

(mPf) $f \circ g=g^{\prime} \circ f$, (i.e., $f$ is an $m$-function as in Defintion 6),

(LPf) $f_{i j}^{\prime} \circ f=f \circ f_{i j}$ for all $(i, j) \in[n] \times[m]$.

Remark 2. The condition (mPf) in Definition 8 can be omitted.

Proposition 1. ([27]) Let $\left(X, g,\left\{f_{i j}\right\}_{(i, j) \in[n] \times[m]}\right)$ be a system which satisfies the properties (LP1) to (LP8), and let $D(X)$ be the lattice of all increasing clopen (closed and open) of $X$. Then, the following conditions are equivalent:

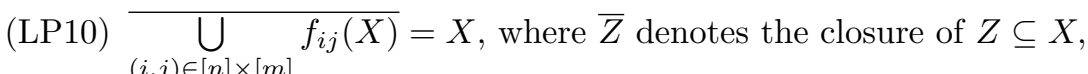

(LP11) if $U, V \in D(X)$ and $f_{i j}^{-1}(U)=f_{i j}^{-1}(V)$ for all $(i, j) \in[n] \times[m]$, then $U=V$,

(LP12) for each $x \in X$, there is $\left(i_{0}, j_{0}\right) \in[n] \times[m]$ such that $f_{i_{0} j_{0}}(x)=x$, (LP13) if $Y, Z \subseteq X$ and $f_{i j}^{-1}(Y)=f_{i j}^{-1}(Z)$ for all $(i, j) \in[n] \times[m]$, then $Y=Z$.

Definition 9. Let $(X, \leq)$ be a partial ordered set. For all $x, y \in X$ such that $x \leq y$, the subset $[x ; y]:=\{z \in X: x \leq z \leq y\}$ is said to be a segment or a closed interval in $X$.

It is worth mentioning the following properties of $L M_{n \times m}$-spaces because they are useful to describe these spaces:

LEMma 3. Let $\left(X, g,\left\{f_{i j}\right\}_{(i, j) \in[n] \times[m]}\right)$ be an $L M_{n \times m}$-space. Then, for any $x \in X$, 
(a) $\left[f_{11}(x) ; f_{(n-1),(m-1)}(x)\right]=\left\{f_{i j}(x):(i, j) \in[n] \times[m]\right\}$,

(b) $x \in\left[f_{11}(x) ; f_{(n-1),(m-1)}(x)\right]$.

Proposition 2. Let $\left(X, g,\left\{f_{i j}\right\}_{(i, j) \in[n] \times[m]}\right)$ be an $L M_{n \times m}$-space. Then $X$ is the cardinal sum of the sets $\left[f_{11}(x) ; f_{(n-1)(m-1)}(x)\right], x \in X$.

Corollary 1. Let $\left(X, g,\left\{f_{i j}\right\}_{(i, j) \in[n] \times[m]}\right)$ be an $L M_{n \times m}$-space. Then it holds that

(LP14) $\min X=\left\{f_{11}(x): x \in X\right\}$,

(LP15) $\max X=\left\{f_{(n-1)(m-1)}(x): x \in X\right\}$.

Corollary 2. Let $\left(X, g,\left\{f_{i j}\right\}_{(i, j) \in[n] \times[m]}\right)$ be an $L M_{n \times m}$-space. Then for any $x \in X$ it holds that

(LP16) $f_{11}(x) \leq x$ and $f_{11}(x)$ is the unique minimal element in $X$ that precedes $x$,

(LP17) $x \leq f_{(n-1)(m-1)}(x)$ and $f_{(n-1)(m-1)}(x)$ is the unique maximal element in $X$ that follows $x$.

Corollary 3. Let $\left(X, g,\left\{f_{i j}\right\}_{(i, j) \in[n] \times[m]}\right)$ be an $L M_{n \times m}$-space. Then, for all interval $I \subseteq X$, the following conditions are equivalent:

(a) $I=\left[f_{11}(x) ; f_{(n-1)(m-1)}(x)\right]$ for some $x \in X$,

(b) $I$ is a maximal interval in $X$.

In addition, in [27], the following results were established:

- If $\left(X, g,\left\{f_{i j}\right\}_{i \in[n] \times[m]}\right)$ is an $L M_{n \times m}$-space. Then,

$$
\left(D(X), \sim_{g},\left\{\sigma_{i j}^{X}\right\}_{i \in[n] \times[m]}\right)
$$

is an $L M_{n \times m}$-algebra, where for every $U \in D(X), \sim_{g} U$ is defined as in $(2.3)$ and

$$
\sigma_{i j}^{X}(U)=f_{i j}^{-1}(U) \text { for all }(i, j) \in[n] \times[m] .
$$

- If $\left(A, \sim,\left\{\sigma_{i j}\right\}_{i \in[n] \times[m]}\right)$ is an $L M_{n \times m}$-algebra and $X(A)$ is the Priestley space associated with $A$, then $\left(X(A), g_{A},\left\{f_{i j}^{A}\right\}_{i \in[n] \times[m]}\right)$ is an $L M_{n \times m}$-space, where for every $S \in X(A), g_{A}(S)$ is defined as $(2.5)$ and

$$
f_{i j}^{A}(S)=\sigma_{i j}^{-1}(S) \text { for all }(i, j) \in[n] \times[m] .
$$


- $\left(A, \sim,\left\{\sigma_{i j}\right\}_{(i, j) \in[n] \times[m]}\right) \cong\left(D(X(A)), \sim,\left\{\sigma_{i j}^{X(A)}\right\}_{(i, j) \in[n] \times[m]}\right)$ and

- $\left(X, g,\left\{f_{i j}\right\}_{(i, j) \in[n] \times[m]}\right) \cong\left(X(D(X)), g_{D(X)},\left\{f_{i j}^{D(X)}\right\}_{(i, j) \in[n] \times[m]}\right)$, via the natural isomorphisms denoted by $\sigma_{A}$ and $\varepsilon_{X}$ respectively, which are defined as in (2.4) and (2.8), respectively.

- The correspondences between the morphisms of both categories are defined in the usual way as in (2.9) and (2.10).

Then, from these results it was concluded that the category $\mathbf{L M}_{\mathbf{n} \times \mathbf{m}} \mathbf{P}$ is dually equivalent to the category $\mathbf{L M}_{\mathbf{n} \times \mathbf{m}} \mathbf{A}$ of $L M_{n \times m}$-algebras and $L M_{n \times m}$-homomorphisms. Moreover, this duality was taken into account to characterize the congruence lattice on an $L M_{n \times m}$-algebra as is indicated in Theorem 2. In order to obtain this characterization the modal subsets of the $L M_{n \times m}$-spaces were taken into account, which we mention below:

Definition 10. Let $\left(X, g,\left\{f_{i j}\right\}_{(i, j) \in[n] \times[m]}\right)$ be an $L M_{n}$-space. A subset $Y$ of $X$ is modal if $Y=f_{i}^{-1}(Y)$ for all $(i, j) \in[n] \times[m]$.

THEOREM 2. ([27]) Let $\left(A, \sim,\left\{\sigma_{i j}\right\}_{(i, j) \in[n] \times[m]}\right)$ be an $L M_{n \times m}$-algebra and $\left(X(A), g_{A},\left\{f_{i j}^{A}\right\}_{(i, j) \in[n] \times[m]}\right)$ be the $L M_{n \times m}$-space associated with $A$. Then, the lattice $C_{M}(X(A))$ of all modal and closed subsets of $X(A)$ is antiisomorphic to the lattice $\operatorname{Con}_{L M_{n \times m}}(A)$ of $L M_{n \times m}$-congruences on $A$, and the anti-isomorphism is the function $\Theta_{M}: C_{M}(X(A)) \longrightarrow \operatorname{Con}_{L M_{n \times m}}(A)$ defined by the same prescription in (2.11).

The previous results allow us to prove the following theorem.

ThEOREM 3. ([27]) Let $\left(X, g,\left\{f_{i j}\right\}_{(i, j) \in[n] \times[m]}\right)$ be an $L M_{n \times m}$-space and let $\left(D(X), \sim_{g},\left\{\sigma_{i j}^{X}\right\}_{(i, j) \in[n] \times[m]}\right)$ be the $L M_{n \times m}$-algebra associated with $X$. Then the following conditions are equivalent:

(a) $X=\left[f_{11}(x), f_{(n-1)(m-1)}(x)\right]$ for all $x \in X$,

(b) $\left(D(X), \sim_{g},\left\{\sigma_{i j}^{X}\right\}_{(i, j) \in[n] \times[m]}\right)$ is a simple $L M_{n \times m}$-algebra,

(c) $\left(D(X), \sim_{g},\left\{\sigma_{i j}^{X}\right\}_{(i, j) \in[n] \times[m]}\right)$ is a subdirectly irreducible $L M_{n \times m^{-}}$ algebra,

(d) $D(X)$ is finite and $D(X) \backslash\{\emptyset, X\}$ has least and greatest element. 


\subsection{Tense $n \times m$-valued Łukasiewicz-Moisil algebras}

In [17], A. V. Figallo and G. Pelaitay introduce the following notion:

Definition 11. An algebra $\left\langle A, \vee, \wedge, \sim,\left\{\sigma_{i j}\right\}_{(i, j) \in[n] \times[m]}, G, H, 0,1\right\rangle$ is a tense $n \times m$-valued Łukasiewicz-Moisil algebra (or tense $L M_{n \times m}$-algebra) if $\left\langle A, \vee, \wedge, \sim,\left\{\sigma_{i j}\right\}_{(i, j) \in[n] \times[m]}, 0,1\right\rangle$, is an $L M_{n \times m}$-algebra and $G, H$ are two unary operators on $A$ which satisfy the following properties:

(T1) $G(1)=1$ and $H(1)=1$,

(T2) $G(x \wedge y)=G(x) \wedge G(y)$ and $H(x \wedge y)=H(x) \wedge H(y)$,

(T3) $G \sigma_{i j}(x)=\sigma_{i j} G(x)$ and $H \sigma_{i j}(x)=\sigma_{i j} H(x)$,

(T4) $x \leq G P(x)$ and $x \leq H F(x)$, where $P(x)=\sim H(\sim x)$ and $F(x)=\sim$ $G(\sim x)$, for any $x, y \in X$ and $(i, j) \in[n] \times[m]$.

A tense $L M_{n \times m}$-algebra $\left\langle A, \vee, \wedge, \sim,\left\{\sigma_{i j}\right\}_{(i, j) \in[n] \times[m]}, G, H, 0,1\right\rangle$ will be denoted in the rest of this paper by $(A, G, H)$ or by $\left(A, \sim,\left\{\sigma_{i j}\right\}_{(i, j) \in[n] \times[m]}, G, H\right)$.

The following lemma contains properties of tense $L M_{n}$-algebras that are useful in what follows.

LEMMA 4. ([27]) The following properties hold in every tense $L M_{n \times m^{-}}$ algebra $(A, G, H)$ :

(T5) $x \leq y$ implies $G(x) \leq G(y)$ and $H(x) \leq H(y)$,

(T6) $x \leq y$ implies $F(x) \leq F(y)$ and $P(x) \leq P(y)$,

(T7) $F(0)=0$ and $P(0)=0$,

(T8) $F(x \vee y)=F(x) \vee F(y)$ and $P(x \vee y)=P(x) \vee P(y)$,

(T9) $P G(x) \leq x$ and $F H(x) \leq x$,

(T10) $G P(x) \wedge F(y) \leq F(P(x) \wedge y)$ and $H F(x) \wedge P(y) \leq P(F(x) \wedge y)$,

(T11) $G(x) \wedge F(y) \leq F(x \wedge y)$ and $H(x) \wedge P(y) \leq P(x \wedge y)$,

$(\mathrm{T} 12) G(x \vee y) \leq G(x) \vee F(y)$ and $H(x \vee y) \leq H(x) \vee P(y)$, for any $x, y \in X$.

Definition 12. ([27]) If $(A, G, H)$ and $\left(A^{\prime}, G^{\prime}, H^{\prime}\right)$ are two tense $L M_{n \times m^{-}}$ algebras, then a morphism of tense $L M_{n \times m}$-algebras $f:(A, G, H) \longrightarrow$ $\left(A^{\prime}, G^{\prime}, H^{\prime}\right)$ is a morphism of $L M_{n \times m}$-algebras such that

(tf) $f(G(a))=G^{\prime}(f(a))$ and $f(H(a))=H^{\prime}(f(a))$, for any $a \in A$. 
Lemma 5. ([27]) Let $(A, G, H)$ be a tense $L M_{n \times m}$-algebra and let $C(A):=$ $\{a \in A: d(a)=a\}$. Then, $\left\langle C(A), \vee, \wedge, \sim,\left\{\sigma_{i j}\right\}_{(i, j) \in[n] \times[m]}, 0,1\right\rangle$ is an $L M_{n \times m}$-algebra.

\section{Topological duality for tense $L M_{n \times m}$-algebras}

In this section, we will develop a topological duality for tense $n \times m$-valued Łukasiewicz-Moisil algebras, taking into account the results established by A. V. Figallo, I. Pascual and G. Pelaitay in [27] and the results obtained by A. V. Figallo and G. Pelaitay in [16]. In order to determine this duality, we introduce a topological category whose objects and their corresponding morphisms are described below.

Definition 13. A system $\left(X, g,\left\{f_{i j}\right\}_{i \in[n] \times[m]}, R\right)$ is a tense $L M_{n \times m}$-space if the following conditions are satisfied:

(i) $\left(X, g,\left\{f_{i j}\right\}_{(i, j) \in[n] \times[m]}\right)$ is an $L M_{n \times m}$-space (Definition 7),

(ii) $R$ is a binary relation on $X$ and $R^{-1}$ is the converse of $R$ such that:

$(\mathrm{tS} 1)(x, y) \in R$ implies $(g(x), g(y)) \in R$,

(tS2) for each $x \in X, R(x)$ and $R^{-1}(x)$ are closed subsets of $X$,

(tS3) for each $x \in X, R(x)=\downarrow R(x) \cap \uparrow R(x)$,

(tS4) $(x, y) \in R$ implies $\left(f_{i j}(x), f_{i j}(y)\right) \in R$ for any $(i, j) \in[n] \times[m]$,

(tS5) $\left(f_{i j}(x), y\right) \in R,(i, j) \in[n] \times[m]$, implies that there exists $z \in X$ such that $(x, z) \in R$ and $f_{i j}(z) \leq y$,

(tS6) $\left(y, f_{i j}(x)\right) \in R,(i, j) \in[n] \times[m]$, implies that there exists $z \in X$ such that $(z, x) \in R$ and $f_{i j}(z) \leq y$,

(tS7) for each $U \in D(X), G_{R}(U), H_{R^{-1}}(U) \in D(X)$, where $G_{R}$ and $H_{R^{-1}}$ are operators on $P(X)$ defined as in (2.1) and (2.2), respectively.

Definition 14. A tense $L M_{n \times m}$-function $f$ from a tense $L M_{n \times m}$-space $\left(X_{1}, g_{1},\left\{f_{i j}^{1}\right\}_{(i, j) \in[n] \times[m]}, R_{1}\right)$ into another one, $\left(X_{2}, g_{2},\left\{f_{i j}^{2}\right\}_{(i, j) \in[n] \times[m]}, R_{2}\right)$ is a function $f: X_{1} \longrightarrow X_{2}$ such that:

(i) $f: X_{1} \longrightarrow X_{2}$ is an $L M_{n \times m}$-function (Definition 8),

(ii) $f: X_{1} \longrightarrow X_{2}$ satisfies the following conditions, for all $x \in X_{1}$ :

(tf1) $f\left(R_{1}(x)\right) \subseteq R_{2}(f(x))$ and $f\left(R_{1}^{-1}(x)\right) \subseteq R_{2}^{-1}(f(x))$,

(tf2) $R_{2}(f(x)) \subseteq \uparrow f\left(R_{1}(x)\right)$,

(tf3) $R_{2}{ }^{-1}(f(x)) \subseteq \uparrow f\left(R_{1}^{-1}(x)\right)$. 
The category that has tense $L M_{n \times m}$-spaces as objects and tense $L M_{n \times m}$-functions as morphisms will be denoted by $\mathbf{t L M}_{\mathbf{n} \times \mathbf{m}} \mathbf{S}$, and $\mathbf{t L M}_{\mathbf{n} \times \mathbf{m}} \mathbf{A}$ will denote the category of tense $L M_{n \times m}$-algebras and tense $L M_{n \times m}$-homomorphisms. Our next task will be to determine that the category $\mathbf{t L M}_{\mathbf{n} \times \mathbf{m}} \mathbf{S}$ is naturally equivalent to the dual category of $\mathbf{t} \mathbf{L} \mathbf{M}_{\mathbf{n} \times \mathbf{m}} \mathbf{A}$.

Now we will show a characterization of tense $L M_{n \times m}$-functions which will be useful later.

Lemma 6. Let $\left(X_{1}, g_{1},\left\{f_{i j}^{1}\right\}_{i \in[n] \times[m]}, R_{1}\right)$ and $\left(X_{2}, g_{2},\left\{f_{i j}^{2}\right\}_{i \in[n] \times[m]}, R_{2}\right)$ be two tense $L M_{n \times m}$-spaces and

$f: X_{1} \longrightarrow X_{2}$ be a tense $L M_{n \times m}$-function. Then, $f$ satisfies the following conditions:

(tf4) $\uparrow f\left(R_{1}(x)\right)=\uparrow R_{2}(f(x))$,

(tf5) $\uparrow f\left(R_{1}^{-1}(x)\right)=\uparrow R_{2}^{-1}(f(x))$, for any $x \in X$.

PROOF: It can be proved using a similar technique to that used in the proof of Lemma 3.4 in [14].

Lemma 7. Let $\left(X, g,\left\{f_{i j}\right\}_{(i, j) \in[n] \times[m]}, R\right)$ be a tense $L M_{n \times m}$-space. Then for all $x, y \in X$ such that $(x, y) \notin R$, the following conditions are satisfied:

(i) There is $U \in D(X)$ such that $y \notin U$ and $x \in G_{R}(U)$ or $y \in U$ and $x \notin F_{R}(U)$, where $F_{R}(U):=\{x \in X: R(x) \cap U \neq \emptyset\}$.

(ii) There is $V \in D(X)$ such that $y \notin V$ and $x \in H_{R^{-1}}(V)$ or $y \in V$ and $x \notin P_{R^{-1}}(V)$, where $P_{R^{-1}}(V):=\left\{x \in X: R^{-1}(x) \cap V \neq \emptyset\right\}$.

Proof: It can be proved in a similar way to Lemma 3.5 of [14].

Lemma 8. Let $\left(X_{1}, g_{1},\left\{f_{i j}^{1}\right\}_{(i, j) \in[n] \times[m]}, R_{1}\right)$ and $\left(X_{2}, g_{2},\left\{f_{i j}^{2}\right\}_{(i, j) \in[n] \times[m]}, R_{2}\right)$ be two tense $L M_{n \times m}$-spaces. Then, the following conditions are equivalent:

(i) $f: X_{1} \longrightarrow X_{2}$ is a tense $L M_{n \times m}$-function,

(ii) $f: X_{1} \longrightarrow X_{2}$ is an $L M_{n \times m}$-function such that, for any $U \in D\left(X_{2}\right)$ : (tf6) $f^{-1}\left(G_{R_{2}}(U)\right)=G_{R_{1}}\left(f^{-1}(U)\right)$, (tf7) $f^{-1}\left(H_{R_{2}^{-1}}(U)\right)=H_{R_{1}^{-1}}\left(f^{-1}(U)\right)$.

ProOF: The proof is similar in spirit to Lemma 3.6 of [14].

Lemma 9 and Corollary 4 can be proved in a similar way to Lemma 3.8 and Corollary 3.9, respectively of [14]. 
Lemma 9. Let $\left(X, g,\left\{f_{i j}\right\}_{(i, j) \in[n] \times[m]}, R\right)$ be a tense $L M_{n \times m}$-space. Then, the following conditions are satisfied for any $x, y, \in X$ and $(i, j) \in[n] \times[m]$ :

(tS11) $R(g(x))=g(R(x)), R^{-1}(g(x))=g\left(R^{-1}(x)\right)$,

$(\mathrm{tS} 12) R\left(f_{i j}(x)\right) \subseteq \bigcup_{y \in R\left(f_{i j}(x)\right)} \uparrow f_{i j}(y)$,

$(\mathrm{tS} 13) R^{-1}\left(f_{i j}(x)\right) \subseteq \bigcup_{y \in R^{-1}\left(f_{i j}(x)\right)} \uparrow f_{i j}(y)$,

$(\mathrm{tS} 14) \uparrow f_{i j}\left(R_{1}(x)\right)=\uparrow R_{2}(f(x))$,

$(\mathrm{tS} 15) \uparrow f_{i j}\left(R_{1}^{-1}(x)\right)=\uparrow R_{2}^{-1}(f(x))$,

(tS16) $f_{i j}^{-1}\left(G_{R}(U)\right)=G_{R}\left(f_{i j}^{-1}(U)\right)$,

(tS17) $f_{i j}^{-1}\left(H_{R^{-1}}(U)\right)=H_{R^{-1}}\left(f_{i j}^{-1}(U)\right)$,

$(\mathrm{tS} 18) f_{i j}^{-1}\left(\sim_{g} U\right)=\sim_{g}\left(f_{(n-i)(m-j)}^{-1}(U)\right)$,

$(\mathrm{tS} 19) f_{i j}^{-1}\left(F_{R}(U)\right)=F_{R}\left(f_{i j}^{-1}(U)\right)$,

$(\mathrm{tS} 20) f_{i j}^{-1}\left(P_{R^{-1}}(U)\right)=P_{R^{-1}}\left(f_{i j}^{-1}(U)\right)$.

Corollary 4. Let $\left(X, g,\left\{f_{i j}\right\}_{(i, j) \in[n] \times[m]}\right)$ be a tense $L M_{n \times m}$-space. Then, the conditions (tS4), (tS5) and (tS6) can be replaced by the following conditions:

$(\mathrm{tS} 16) f_{i j}^{-1}\left(G_{R}(U)\right)=G_{R}\left(f_{i j}^{-1}(U)\right)$ for any $U \in D(X)$

$(\mathrm{tS} 17) f_{i j}^{-1}\left(H_{R^{-1}}(U)\right)=H_{R^{-1}}\left(f_{i j}^{-1}(U)\right)$ for any $U \in D(X)$.

Next, we will define a contravariant functor from $\mathbf{t L M}_{\mathbf{n} \times \mathbf{m}} \mathbf{S}$ to $\mathbf{t L M}_{\mathbf{n} \times \mathbf{m}} \mathbf{A}$.

Lemma 10. Let $\left(X, g,\left\{f_{i j}\right\}_{(i, j) \in[n] \times[m]}, R\right)$ be a tense $L M_{n \times m}$-space. Then,

$$
\Psi(X)=\left\langle D(X), \sim_{g},\left\{\sigma_{i j}^{X}\right\}_{(i, j) \in[n] \times[m]}, G_{R}, H_{R^{-1}}, \emptyset, X\right\rangle
$$

is a tense $L M_{n \times m}$-algebra, where for all $U \in D(X), \sim_{g} U, \sigma_{i j}^{X}(U),(i, j) \in$ $[n] \times[m], G_{R}(U)$ and $H_{R^{-1}}(U)$ are defined as in (2.3), (2.14), (2.1) and (2.2), respectively.

Proof: From [27] and [16, Lemma 4.3] it follows that the reduct $\langle D(X)$, $\left.\sim_{g},\left\{\sigma_{i j}^{X}\right\}_{(i, j) \in[n] \times[m]}, \emptyset, X\right\rangle$ is an $L M_{n \times m}$-algebra and the structure $\langle D(X)$, $\left.\sim_{g}, G_{R}, H_{R^{-1}}, \emptyset, X\right\rangle$ is a tense De Morgan algebra, respectively. Therefore, the properties (T1), (T2) and (T4) of tense $L M_{n \times m}$-algebras (Definition 11) hold. In addition, since any $U \in D(X)$ satisfies properties (tS16) and (tS17) in Lemma 9, then we can assert that property (T3) holds too, and so the proof is complete. 
LEMma 11. Let $f:\left(X_{1}, g_{1},\left\{f_{i j}^{1}\right\}_{(i, j) \in[n] \times[m]}\right) \longrightarrow\left(X_{2}, g_{2},\left\{f_{i}^{2}\right\}_{(i, j) \in[n] \times[m]}\right)$ be a morphism of tense $L M_{n \times m}$-spaces. Then, the map $\Psi(f): D\left(X_{2}\right) \longrightarrow$ $D\left(X_{1}\right)$ defined by $\Psi(f)(U)=f^{-1}(U)$ for all $U \in D\left(X_{2}\right)$, is a tense $L M_{n \times m}$-homomorphism.

Proof: It follows from the results established in [27] and Lemma 8.

The previous two lemmas show that $\Psi$ is a contravariant functor from $\mathbf{t L M}_{\mathbf{n}} \mathbf{S}$ to $\mathbf{t} \mathbf{L} \mathbf{M}_{\mathbf{n}} \mathbf{A}$. To achieve our goal we need to define a contravariant functor from $\mathbf{t} \mathbf{L} \mathbf{M}_{\mathbf{n}} \mathbf{A}$ to $\mathbf{t} \mathbf{L M} \mathbf{n} \mathbf{S}$.

Lemma 12. Let $(A, G, H)$ be a tense $L M_{n \times m}$-algebra and let $S, T \in X(A)$. Then the following conditions are equivalent:

(i) $G^{-1}(S) \subseteq T \subseteq F^{-1}(S)$,

(ii) $H^{-1}(T) \subseteq S \subseteq P^{-1}(T)$.

Proof: In a similar way to [18, Lemma 3.8].

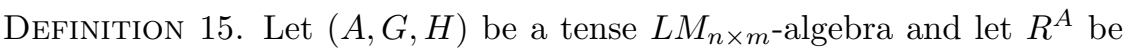
the relation defined on $X(A)$ by the prescription:

$$
(S, T) \in R^{A} \Longleftrightarrow G^{-1}(S) \subseteq T \subseteq F^{-1}(S) .
$$

Remark 3. Lemma 12 means that we have two ways to define the relation $R^{A}$, either by using $G$ and $F$, or by using $H$ and $P$.

The following lemma, whose proof can be obtained as in [18, Lemma 3.11], will be essential for the proof of Lemma 14 .

Lemma 13. Let $(A, G, H)$ be a tense $L M_{n \times m}$-algebra and let $S \in X(A)$ and $a \in A$. Then,

(i) $G(a) \notin S$ if and only if there exists $T \in X(A)$ such that $(S, T) \in R^{A}$ and $a \notin T$,

(ii) $H(a) \notin S$ if and only if there exists $T \in X(A)$ such that $(S, T) \in$ $R^{A^{-1}}$ and $a \notin T$.

Lemma 14. Let $(A, G, H)$ be an $L M_{n \times m}$-algebra and $X(A)$ be the Priestley space associated with $A$. Then, $\Phi(A)=\left(X(A), g_{A},\left\{f_{i j}^{A}\right\}_{(i, j) \in[n] \times[m]}, R^{A}\right)$ is a tense $L M_{n \times m}$-space, where for every $S \in X(A), g_{A}(S)$ and $f_{i j}^{A}(S)$ are defined as in (2.5) and (2.15), respectively and $R^{A}$ is the relation defined on $X(A)$ as in (3.1). Besides, $\sigma_{A}: A \longrightarrow D(X(A))$, defined by the prescription (2.4), is a tense $L M_{n \times m}$-isomorphism. 
Proof: From [27] and [16, Lemma 5.6] it follows that the system $\left(X(A), g_{A},\left\{f_{i j}^{A}\right\}_{(i, j) \in[n] \times[m]}\right)$ is an $L M_{n \times m}$-space and $\left(X(A), g_{A}, R^{A}, R^{A^{-1}}\right)$ is a tense $m P$-space, and so properties (tS1), (tS2) (tS3) and (tS7) of tense $L M_{n \times m}$-spaces hold (Definition 13). Also, from Corollary 4 we have that the conditions (tS4), (tS5) and (tS6) are satisfied. Therefore, we have that $\left(X(A), g_{A},\left\{f_{i j}^{A}\right\}_{(i, j) \in[n] \times[m]}, R^{A}\right)$ is a tense $L M_{n \times m}$-space. In addi-

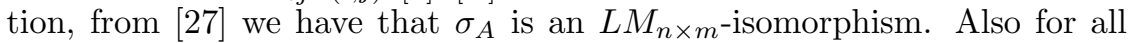
$a \in A, G_{R^{A}}\left(\sigma_{A}(a)\right)=\sigma_{A}(G(a))$ and $H_{R^{A-1}}\left(\sigma_{A}(a)\right)=\sigma_{A}(H(a))$. Indeed, let us take a prime filter $S$ such that $G(a) \notin S$. By Lemma 13, there exists $T \in X(A)$ such that $(S, T) \in R^{A}$ and $a \notin T$. Then, $R^{A}(S) \nsubseteq \sigma_{A}(a)$. So, $S \notin G_{R^{A}}\left(\sigma_{A}(a)\right)$ and, therefore, $G_{R^{A}}(\sigma(a)) \subseteq \sigma_{A}(G(a))$. Moreover, it is immediate that $\sigma_{A}(G(a)) \subseteq G_{R_{A}}\left(\sigma_{A}(a)\right)$. Similarly we obtain that $H_{R^{A^{-1}}}\left(\sigma_{A}(a)\right)=\sigma_{A}(H(a))$ and so $\sigma_{A}$ is a tense $L M_{n \times m^{-}}$isomorphism.

Lemma 15. Let $\left(A_{1}, G_{1}, H_{1}\right)$ and $\left(A_{2}, G_{2}, H_{2}\right)$ be two $L M_{n \times m}$-algebras and $h: A_{1} \longrightarrow A_{2}$ be a tense $L M_{n \times m}$-homomorphism. Then, the map $\Phi(h)$ : $X\left(A_{2}\right) \longrightarrow X\left(A_{1}\right)$, defined by $\Phi(h)(S)=h^{-1}(S)$ for all $S \in X\left(A_{2}\right)$, is a tense $L M_{n \times m}$-function.

Proof: It follows from the results established in [27] and [16, Lemma 5.7].

Lemmas 14 and 15 show that $\Phi$ is a contravariant functor from $\mathbf{t L M}_{\mathbf{n} \times \mathbf{m}} \mathbf{A}$ to $\mathbf{t L M}_{\mathbf{n} \times \mathbf{m}} \mathbf{S}$.

The following characterization of isomorphisms in the category $\mathbf{t L M}_{\mathbf{n} \times \mathbf{m}} \mathbf{S}$ will be used to determine the duality that we set out to prove. Proposition 3 . Let $\left(X_{1}, g_{1},\left\{f_{i j}^{1}\right\}_{(i, j) \in[n] \times[m]}, R_{1}\right)$ and $\left(X_{2}\right.$, $\left.g_{2},\left\{f_{i j}^{2}\right\}_{(i, j) \in[n] \times[m]}, R_{2}\right)$ be two tense $L M_{n \times m}$-spaces. Then, the following conditions are equivalent, for every function $f: X_{1} \longrightarrow X_{2}$ :

(i) $f$ is an isomorphism in the category $\operatorname{tLM}_{\mathbf{n}} \mathbf{S}$,

(ii) $f$ is a bijective $L M_{n \times m}$-function such that for all $x, y \in X_{1}$ :

(itf) $(x, y) \in R_{1} \Longleftrightarrow(f(x), f(y)) \in R_{2}$.

Proof: It is routine.

The map $\varepsilon_{X}: X \longrightarrow X(D(X))$, defined as in (2.8), leads to another characterization of tense $L M_{n \times m}$-spaces, which also allow us to assert that this map is an isomorphism in the category $\mathbf{t} \mathbf{L M}_{\mathbf{n} \times \mathbf{m}} \mathbf{S}$, as we will describe below: 
Lemma 16. Let $\left(X, g,\left\{f_{i j}\right\}_{(i, j) \in[n] \times[m]}, R\right)$ be a tense $L M_{n \times m}$-space, $\varepsilon_{X}$ : $X \longrightarrow X(D(X))$ be the map defined by the prescription (2.8) and let $R^{D(X)}$ be the relation defined on $X(D(X))$ by means of the operators $G_{R}$ and $F_{R}$ as follows:

$$
\left(\varepsilon_{X}(x), \varepsilon_{X}(y)\right) \in R^{D(X)} \Longleftrightarrow G_{R}^{-1}\left(\varepsilon_{X}(x)\right) \subseteq \varepsilon_{X}(y) \subseteq F_{R}^{-1}\left(\varepsilon_{X}(x)\right) .
$$

Then, the following property holds:

(tS5) $(x, y) \in R$ implies $\left(\varepsilon_{X}(x), \varepsilon_{X}(y)\right) \in R^{D(X)}$.

PROOF: It is routine.

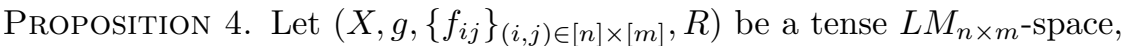
$\varepsilon_{X}: X \longrightarrow X(D(X))$ be the function defined by the prescription (2.8) and let $R^{D(X)}$ be the relation defined on $X(D(X))$ by the prescription (3.2). Then, the condition (tS3) can be replaced by the following one:

$(\mathrm{tS} 18)\left(\varepsilon_{X}(x), \varepsilon_{X}(y)\right) \in R^{D(X)} \Longleftrightarrow(x, y) \in R$.

Proof: It can be proved in a similar way to [16, Proposition 5.5].

Corollary 5. Let $\left(X, g,\left\{f_{i j}\right\}_{(i, j) \in[n] \times[m]}, R\right)$ be a tense $L M_{n \times m}$-space. Then, the map $\varepsilon_{X}: X \longrightarrow X(D(X))$ is an isomorphism in the category $\mathbf{t L M}_{\mathbf{n} \times \mathbf{m}} \mathbf{S}$.

PRoOF: It follows from the results established in [27], Lemma 16, Propositions 3 and 4 .

Then, from the above results and using the usual procedures we can prove that the functors $\Phi \circ \Psi$ and $\Psi \circ \Phi$ are naturally equivalent to the identity functors on $\mathbf{t} \mathbf{L} \mathbf{M}_{\mathbf{n} \times \mathbf{m}} \mathbf{S}$ and $\mathbf{t} \mathbf{L} \mathbf{M}_{\mathbf{n} \times \mathbf{m}} \mathbf{A}$, respectively, from which we conclude:

THEOREM 4. The category $\mathbf{t} \mathbf{L M}_{\mathbf{n} \times \mathbf{m}} \mathbf{S}$ is naturally equivalent to the dual of the category $\mathbf{t} \mathbf{L} \mathbf{M}_{\mathbf{n}} \mathbf{A}$.

\section{Subdirectly irreducible tense $\mathbf{L M}_{\mathbf{n} \times \mathbf{m}}$-algebras}

In this section, our first objective is the characterization of the congruence lattice on a tense $L M_{n \times m}$-algebra by means of certain closed and modal subsets of its associated tense $L M_{n \times m}$-space. Later, this result will be taken into account to characterize simple and subdirectly irreducible tense 
$L M_{n \times m}$-algebras. With this purpose, we will start by introducing the following notion.

Definition 16. Let $\left(X, g,\left\{f_{i j}\right\}_{(i, j) \in[n] \times[m]}, R\right)$ be a tense $L M_{n \times m}$-space. A subset $Y$ of $X$ is a tense subset if it satisfies the following conditions for all $y, z \in X$ :

(ts1) if $y \in Y$ and $z \in R(y)$, then there is $w \in Y$ such that $w \in R(y) \cap \downarrow z$, (ts2) if $y \in Y$ and $z \in R^{-1}(y)$, then there is $v \in Y$ such that $v \in R^{-1}(y) \cap \downarrow z$.

In [27] the following characterizations of a modal subset of an $L M_{n \times m^{-}}$ space were obtained.

Proposition 5. ([27]) Let $\left(X, g,\left\{f_{i j}\right\}_{(i, j) \in[n] \times[m]}\right)$ be an $L M_{n \times m^{-} \text {space }}$ and $Y$ be a nonempty subset of $X$. Then, the following conditions are equivalent:

(a) $Y$ is modal,

(b) $Y$ is involutive and increasing,

(c) $Y=\bigcup_{y \in Y}\left[f_{11}(y), f_{(n-1)(m-1)}(y)\right]$ (i.e. $Y$ is the cardinal sum of certain maximal intervals of $X)$.

Corollary 6. Let $\left(X, g,\left\{f_{i j}\right\}_{(i, j) \in[n] \times[m]}\right)$ be an $L M_{n \times m}$-space. If $\left\{Y_{i}\right\}_{i \in I}$ is a family of modal subsets of $X$, then $\bigcap_{i \in I} Y_{i}$ is a modal subset of $X$.

ProOF: It is a direct consequence of Proposition 5.

The notion of a modal and tense subset of a tense $L M_{n \times m}$-space has several equivalent formulations, which will be useful later:

Proposition 6. Let $\left(X, g,\left\{f_{i j}\right\}_{(i, j) \in[n] \times[m]}, R\right)$ be a tense $L M_{n \times m}$-space. If $Y$ is a modal subset of $X$, then the following conditions are equivalent:

(i) $Y$ is a tense subset,

(ii) for all $y \in Y$, the following conditions are satisfied:

(ts3) $R(y) \subseteq Y$,

(ts4) $R^{-1}(y) \subseteq Y$,

(iii) $Y=G_{R}(Y) \cap Y \cap H_{R^{-1}}(Y)$, where $G_{R}(Y):=\{x \in X: R(x) \subseteq Y\}$ and $H_{R^{-1}}(Y):=\left\{x \in X: R^{-1}(x) \subseteq Y\right\}$.

Proof: (i) $\Rightarrow$ (ii): Let $y \in Y$ and $z \in R(y$ ), then by (i) and (ts1), there is $w \in Y$ such that $w \in R(y)$ and $w \leq z$. Since $Y$ is modal, from Proposition 5 it follows that $z \in Y$ and therefore $R(y) \subseteq Y$. Using an analogous reasoning we get that $R^{-1}(y) \subseteq Y$. 
(ii) $\Rightarrow(\mathrm{i})$ : It is immediate.

(ii) $\Leftrightarrow$ (iii): It is immediate.

The closed, modal and tense subsets of the tense $L M_{n \times m}$-space associated with a tense $L M_{n \times m}$-algebra perform a fundamental roll in the characterization of the tense $L M_{n \times m}$-congruences on these algebras as we will show next.

TheOREM 5. Let $(A, G, H)$ be a tense $L M_{n \times m}$-algebra, and $\left(X(A), g_{A}\right.$, $\left.\left\{f_{i j}^{A}\right\}_{(i, j) \in[n] \times[m]}, R^{A}\right)$ be the tense $L M_{n \times m}$-space associated with $A$. Then, the lattice $C_{M T}(X(A))$ of all closed, modal and tense subsets of $X(A)$ is anti-isomorphic to the lattice $\operatorname{Con}_{t L M_{n \times m}}(A)$ of tense $L M_{n \times m}$-congruences on $A$, and the isomorphism is the function $\Theta_{M T}$ defined by the same prescription as in (2.11).

Proof: It immediately follows from Theorems 1 and 2 and the fact that $C_{M T}(X(A))=C_{M}(X(A)) \cap C_{T}(X(A))$ and for all $\varphi \subseteq A \times A, \varphi \in$ $\operatorname{Con}_{t L M_{n \times m}}(A)$ iff $\varphi$ is both an $L M_{n \times m}$-congruence on $A$ and a tense De Morgan congruence on $A$.

Next, we will use the results already obtained in order to determine the simple and subdirectly irreducible tense $L M_{n \times m}$-algebras.

Corollary 7 . Let $(A, G, H)$ be a tense $L M_{n \times m}$-algebra, and $\left(X(A), g_{A}\right.$, $\left.\left\{f_{i}^{A}\right\}_{(i, j) \in[n] \times[m]}, R^{A}\right)$ be the tense $L M_{n \times m}$-space associated with $A$. Then, the following conditions are equivalent:

(i) $(A, G, H)$ is a simple tense $L M_{n \times m}$-algebra,

(ii) $C_{M T}(X(A))=\{\emptyset, X(A)\}$.

Proof: It is a direct consequence of Theorem 5 .

Corollary 8. Let $(A, G, H)$ be a tense $L M_{n \times m}$-algebra, and $\left(X(A), g_{A}\right.$, $\left.\left\{f_{i j}^{A}\right\}_{(i, j) \in[n] \times[m]}, R^{A}\right)$ be the tense $L M_{n \times m}$-space associated with $A$. Then, the following conditions are equivalent:

(i) $(A, G, H)$ is a subdirectly irreducible tene $L M_{n \times m}$-algebra,

(ii) there is $Y \in C_{M T}(X(A)) \backslash\{X(A)\}$ such that $Z \subseteq Y$ for all $Z \in$ $C_{M T}(X(A)) \backslash\{X(A)\}$

Proof: It is a direct consequence of Theorem 5 . 
Proposition 7. Let $\left(X, g,\left\{f_{i j}\right\}_{(i, j) \in[n] \times[m]}, R\right)$ be a tense $L M_{n \times m}$-space. If $Y$ is a modal subset of $X$, then $G_{R}(Y)$ and $H_{R^{-1}}(Y)$ are also modal.

Proof: Let $Y$ be a modal subset of $X$. From Proposition 2 it follows immediately that $(1) G_{R}(Y) \subseteq \bigcup_{z \in G_{R}(Y)}\left[f_{11}(z), f_{(n-1)(m-1)}(z)\right]$. Let (2) $z \in G_{R}(Y)$ and let $(3) w \in\left[f_{11}(z), f_{(n-1)(m-1)}(z)\right]$, then from (3) and properties (LP5) and (LP6), we obtain that (4) $f_{i j}(w)=f_{r s}(z)$ for all $(i, j),(r, s) \in[n] \times[m]$. Let (5) $t \in R(w)$, then by (4), (5) and property (tS3), we infer that $f_{11}(t) \in R\left(f_{11}(z)\right)$ and therefore, from properties (tS4), (LP5) and (LP6), we can assert that there exists $y \in X$ such that (5) $y \in R(z)$ and $(6) f_{i j}(y)=f_{r s}(t)$ for all $(i, j),(r, s) \in[n] \times[m]$. From $(2)$ and (5) we get that $y \in Y$. Since $Y$ is modal, then from this last assertion and (6) it results that $f_{i j}(t) \in Y$ for all $(i, j) \in[n] \times[m]$. Then, since $Y$ is modal, we have that $t \in Y$, from which we deduce by $(5)$ that $R(w) \subseteq Y$, which allows to assert that $w \in G_{R}(Y)$. Therefore, from (3) we can set that $\bigcup_{z \in G_{R}(Y)}\left[f_{11}(z), f_{(n-1)(m-1)}(z)\right] \subseteq G_{R}(Y)$. Then, from (1) it follows that $G_{R}(Y)=\bigcup_{z \in G_{R}(Y)}\left[f_{11}(z), f_{(n-1)(m-1)}(z)\right]$, and so from Proposition 5, we conclude that $G_{R}(Y)$ is modal. The proof that $H_{R^{-1}}(Y)$ is modal is similar.

The characterization of modal and tense subsets of a tense $L M_{n \times m^{-}}$ space, given in Proposition 6, prompts us to introduce the following definition:

Definition 17. Let $\left(X, g,\left\{f_{i j}\right\}_{(i, j) \in[n] \times[m]}, R\right)$ be a tense $L M_{n \times m}$-space and let $d_{X}: P(X) \longrightarrow P(X)$ defined by:

$$
d_{X}(Z)=G_{R}(Z) \cap Z \cap H_{R^{-1}}(Z) \text {, for all } Z \in P(X) .
$$

For each $n \in \omega$, let $d_{X}^{n}: P(X) \longrightarrow P(X)$, defined by:

$$
d_{X}^{0}(Z)=Z, d_{X}^{n+1}(Z)=d_{X}\left(d_{X}^{n}(Z)\right) \text {, for all } Z \in P(X) .
$$

By using the above functions $d_{X}, d_{X}^{n}, n \in \omega$, we obtain another equivalent formulation of the notion of modal and tense subset of a tense $L M_{n \times m^{-}}$ space.

Lemma 17. Let $\left(X, g,\left\{f_{i j}\right\}_{(i, j) \in[n] \times[m]}, R\right)$ be a tense $L M_{n \times m}$-space. If $Y$ is modal subset of $X$, then the following conditions are equivalent:

(i) $Y$ is a tense subset, 
(ii) $Y=d_{X}^{n}(Y)$, for all $n \in \omega$,

(iii) $Y=\bigcap_{n \in \omega} d_{X}^{n}(Y)$.

PROOF: It is an immediate consequence of Proposition 6 and Definition 17.

Proposition 8. Let $\left(X, g,\left\{f_{i j}\right\}_{(i, j) \in[n] \times[m]}, R\right)$ be a tense $L M_{n \times m}$-space and $\left(D(X), G_{R}, H_{R^{-1}}\right)$ be the tense $L M_{n \times m^{-}}$-algebra associated with $X$. Then, for all $n \in \omega$, for all $U, V \in D(X)$ and for all $(i, j) \in[n] \times[m]$, the following conditions are satisfied:

(d0) $d_{X}^{n}(U) \in D(X)$,

(d1) $d_{X}^{n}(X)=X$ and $d_{X}^{n}(\emptyset)=\emptyset$,

(d2) $d_{X}^{n+1}(U) \subseteq d_{X}^{n}(U)$

(d3) $d_{X}^{n}(U \cap V)=d_{X}^{n}(U) \cap d_{X}^{n}(V)$,

(d4) $U \subseteq V$ implies $d_{X}^{n}(U) \subseteq d_{X}^{n}(V)$,

(d5) $d_{X}^{n}(U) \subseteq U$

$(\mathrm{d} 6) d_{X}^{n+1}(U) \subseteq G_{R}\left(d_{X}^{n}(U)\right)$ and $d_{X}^{n+1}(U) \subseteq H_{R^{-1}}\left(d_{X}^{n}(U)\right)$,

(d7) $d_{X}^{n}\left(f_{i j}^{-1}(U)\right)=f_{i j}^{-1}\left(d_{X}^{n}(U)\right)$ for any $n \in \omega$ and $(i, j) \in[n] \times[m]$,

(d8) if $U$ is modal, then $d_{X}^{n}(U)$ is modal,

(d9) $\cap d_{X}^{n}\left(f_{i j}^{-1}(U)\right)$ is a closed, modal and tense subset of $X$ and therefore $d_{X}\left(\bigcap_{n \in \omega} d_{X}^{n}\left(f_{i j}^{-1}(U)\right)\right)=\bigcap_{n \in \omega} d_{X}^{n}\left(f_{i j}^{-1}(U)\right)$.

Proof: From Definition 17, Lemma 14 and the fact that $G_{R}, H_{R^{-1}}$ and $d_{X}^{n}, n \in \omega$, are monotonic operations it immediately follows that properties $(\mathrm{d} 0),(\mathrm{d} 1),(\mathrm{d} 2),(\mathrm{d} 3),(\mathrm{d} 4),(\mathrm{d} 5)$ and (d6) hold.

(d7): Let $U \in D(X)$ and $(i, j) \in[n] \times[m]$, then $d_{X}\left(f_{i j}^{-1}(U)\right)=f_{i j}^{-1}(U) \cap$ $G_{R}\left(f_{i j}^{-1}(U)\right) \cap H_{R^{-1}}\left(f_{i j}^{-1}(U)\right)$. From the last assertion and properties $(\mathrm{tS} 17)$ and (tS18) in Lemma 9, we infer that $(1) d_{X}\left(f_{i j}^{-1}(U)\right)=f_{i j}^{-1}(U \cap$ $\left.G_{R}(U) \cap H_{R^{-1}}(U)\right)=f_{i j}^{-1}\left(d_{X}(U)\right)$ for any $U \in D(X)$ and $(i, j) \in[n] \times[m]$. Suppose that $d_{X}^{n-1}\left(f_{i j}^{-1}(U)\right)=f_{i j}^{-1}\left(d_{X}^{n-1}(U)\right)$, for any $n \in \omega$ and $(i, j) \in$ $[n] \times[m]$, then $(2) d_{X}^{n}\left(f_{i j}^{-1}(U)\right)=d_{X}\left(d_{X}^{n-1}\left(f_{i j}^{-1}(U)\right)\right)=d_{X}\left(f_{i j}^{-1}\left(d_{X}^{n-1}(U)\right)\right)$. Taking into account that $d_{X}^{n-1}(U) \in D(X)$ and (1), we get that $d_{X}\left(f_{i j}^{-1}\left(d_{X}^{n-1}(U)\right)\right)=f_{i j}^{-1}\left(d_{X}\left(d_{X}^{n-1}(U)\right)\right)=f_{i j}^{-1}\left(d_{X}^{n}(U)\right)$, and so from $(2)$ the proof is complete. 
(d8): It is a direct consequence of Corollary 6 and Proposition 7.

(d9): Let $U \in D(X)$. Then, from Lemma 14 and the prescription (2.14), we have that $f_{i j}^{-1}(U) \in D(X)$. Also, from (LP5), $f_{i j}^{-1}(U)$ is a modal subset of $X$ for all $(i, j) \in[n] \times[m]$, from which it follows by $(\mathrm{d} 7)$ that for $n \in \omega$ and $(i, j) \in[n] \times[m], d_{X}^{n}\left(f_{i j}^{-1}(U)\right)$ is a modal and closed subset of $X$, and so by Corollary 6 and the fact that the arbitrary intersection of closed subsets of $X$ is closed, we get that $\bigcap_{n \in \omega} d_{X}^{n}\left(f_{i j}^{-1}(U)\right)$ is a modal and closed subset of $X$. If $\bigcap_{n \in \omega} d_{X}^{n}\left(f_{i j}^{-1}(U)\right)=\emptyset$, then it is verified that $\bigcap_{n \in \omega} d_{X}^{n}\left(f_{i j}^{-1}(U)\right)$ is a closed, modal and tense subset of $X$. Suppose now that there exists $y \in \bigcap_{n \in \omega} d_{X}^{n}\left(f_{i j}^{-1}(U)\right)$. Since, $f_{i j}^{-1}(U) \in D(X)$ for any $(i, j) \in[n] \times[m]$, then from $(\mathrm{d} 6)$ it follows that $y \in G_{R}\left(d_{X}^{n-1}\left(f_{i j}^{-1}(U)\right)\right)$ and $y \in H_{R^{-1}}\left(d_{X}^{n-1}\left(f_{i j}^{-1}(U)\right)\right)$ for all $n \in \omega$. Therefore, $R(y) \subseteq d_{X}^{n-1}\left(f_{i j}^{-1}(U)\right)$ and $R^{-1}(y) \subseteq d_{X}^{n-1}\left(f_{i j}^{-1}(U)\right)$ for all $n \in \omega$ and consequently $R(y) \subseteq$ $\bigcap_{n \in \omega} d_{X}^{n}\left(f_{i j}^{-1}(U)\right)$ and $R^{-1}(y) \subseteq \bigcap_{n \in \omega} d_{X}^{n}\left(f_{i j}^{-1}(U)\right)$ for all $(i, j) \in[n] \times[m]$. From these last assertions, the fact that $\bigcap_{n \in \omega} d_{X}^{n}\left(f_{i j}^{-1}(U)\right)$ is a modal and closed subset of $X$ and Proposition 8, we have that $\bigcap_{n \in \omega} d_{X}^{n}\left(f_{i j}^{-1}(U)\right)$ is a tense subset, from which we conclude, by Lemma 17, that $d_{X}\left(\bigcap_{n \in \omega} d_{X}^{n}\left(f_{i j}^{-1}(U)\right)\right)=\bigcap_{n \in \omega} d_{X}^{n}\left(f_{i j}^{-1}(U)\right)$.

As consequences of Proposition 8 and the above duality for tense $L M_{n \times m}$-algebras (Lemma 14) we obtain the following corollaries.

Corollary 9. Let $\left(A, \sim,\left\{\sigma_{i j}\right\}_{(i, j) \in[n] \times[m]}, G, H\right)$ be a tense $L M_{n \times m}$-algebra and consider the function $d: A \longrightarrow A$, defined by $d(a)=G(a) \wedge a \wedge$ $H(a)$, for all $a \in A$. For all $n \in \omega$, let $d^{n}: A \longrightarrow A$ be a function, defined by $d^{0}(a)=a$ and $d^{n+1}(a)=d\left(d^{n}(a)\right)$, for all $a \in A$. Then, for all $n \in \omega$ and $a, b \in A$, the following conditions are satisfied:

(d1) $d^{n}(1)=1$ and $d^{n}(0)=0$,

(d2) $d^{n+1}(a) \leq d^{n}(a)$,

(d3) $d^{n}(a \wedge b)=d^{n}(a) \wedge d^{n}(b)$,

(d4) $a \leq b$ implies $d^{n}(a) \leq d^{n}(b)$

(d5) $d^{n}(a) \leq a$,

(d6) $d^{n+1}(a) \leq G\left(d^{n}(a)\right)$ and $d^{n+1}(a) \leq H\left(d^{n}(a)\right)$, 
(d7) for all $(i, j) \in[n] \times[m]$ and $n \in \omega, d^{n}\left(\sigma_{i j}(a)\right)=\sigma_{i j}\left(d^{n}(a)\right)$.

Corollary 10. Let $(A, G, H)$ be a tense $L M_{n \times m}$-algebra, $\left(X(A), g_{A}\right.$,

$\left.\left\{f_{i j}^{A}\right\}_{(i, j) \in[n] \times[m]}, R^{A}\right)$ be the tense $L M_{n \times m}$-space associated with $A$ and let $\sigma_{A}: A \longrightarrow D(X(A))$ be the map defined by the prescription (2.4). Then, $\sigma_{A}\left(d^{n}(a)\right)=d_{X(A)}^{n}\left(\sigma_{A}(a)\right)$ for all $a \in A$ and $n \in \omega$.

Proof: It is a direct consequence of Lemma 14.

It seems worth mentioning that the operator $d$ defined in Corollary 9 was previously defined in [19] for tense algebras, in [12] for tense $M V$ algebras, and in $[8,9]$ for tense $\theta$-valued Łukasiewicz-Moisil algebras, respectively.

LEMMA 18. Let $(A, G, H)$ be a tense $L M_{n \times m}$-algebra. If $\bigwedge_{i \in I} a_{i}$ exists, then the following conditions hold:

(i) $\bigwedge_{i \in I} G\left(a_{i}\right)$ exists and $\bigwedge_{i \in I} G\left(a_{i}\right)=G\left(\bigwedge_{i \in I} a_{i}\right)$,

(ii) $\bigwedge_{i \in I} H\left(a_{i}\right)$ exists and $\bigwedge_{i \in I} H\left(a_{i}\right)=H\left(\bigwedge_{i \in I} a_{i}\right)$,

(iii) $\bigwedge_{i \in I} d\left(a_{i}\right)$ exists and $\bigwedge_{i \in I} d^{n}\left(a_{i}\right)=d^{n}\left(\bigwedge_{i \in I} a_{i}\right)$ for all $n \in \omega$.

Proof:

(i): Assume that $a_{i} \in A$ for all $i \in I$ and $\bigwedge_{i \in I} a_{i}$ exists. Since $\bigwedge_{i \in I} a_{i} \leq a_{i}$, we have by (T2) that $G\left(\bigwedge_{i \in I} a_{i}\right) \leq G\left(a_{i}\right)$ for each $i \in I$. Thus, $G\left(\bigwedge_{i \in I} a_{i}\right)$ is a lower bound of the set $\left\{G\left(a_{i}\right): i \in I\right\}$. Assume now that $b$ is a lower bound of the set $\left\{G\left(a_{i}\right): i \in I\right\}$. From (T5) and (T6) we have that $P(b) \leq P G\left(a_{i}\right) \leq a_{i}$ for each $i \in I$. So, $P(b) \leq \bigwedge_{i \in I} a_{i}$. Besides, the pair $(G, P)$ is a Galois connection, this means that $x \leq G(y) \Longleftrightarrow P(x) \leq y$, for all $x, y \in A$. So, we can infer that $b \leq G\left(\bigwedge_{i \in I} a_{i}\right)$. This proves that $\bigwedge_{i \in I} G\left(a_{i}\right)$ exists and $\bigwedge_{i \in I} G\left(a_{i}\right)=G\left(\bigwedge_{i \in I} a_{i}\right)$.

(ii): The proof for the operator $H$ is analogous to the proof for $G$.

(iii): It is a direct consequence of (i) and (ii).

For invariance properties we have:

Lemma 19. Let $\left(X, g,\left\{f_{i j}\right\}_{(i, j) \in[n] \times[m]}, R\right)$ be a tense $L M_{n \times m}$-space and $\left(D(X), G_{R}, H_{R^{-1}}\right)$ be the tense $L M_{n \times m^{-}}$-algebra associated with $X$. Then, 
for all $U, V, W \in D(X)$ such that $U=d_{X}(U), V=d_{X}(V)$ and for some $\left(i_{0}, j_{0}\right) \in[n] \times[m], d_{X}\left(f_{i_{0} j_{0}}^{-1}(W)\right)=f_{i_{0} j_{0}}^{-1}(W)$, the following properties are satisfied:

(i) $U \cap V=d_{X}(U \cap V)$,

(ii) $U \cup V=d_{X}(U \cup V)$,

(iii) $\sim_{g} U=d_{X}\left(\sim_{g} U\right)$,

(iv) $d_{X}\left(f_{i j}^{-1}(W)\right)=f_{i j}^{-1}(W)$ for all $(i, j) \in[n] \times[m]$.

\section{ProOF:}

(i): It immediately follows from the definition of the function $d_{X}$ and property (T2) of tense $L M_{n \times m}$-algebras.

(ii): Taking into account that $U=d_{X}(U)$ and $V=d_{X}(V)$ and the fact that the operations $G_{R}$ and $H_{R^{-1}}$ are increasing, we infer that $U \cup V \subseteq$ $G_{R}(U \cup V)$ and $U \cup V \subseteq H_{R^{-1}}(U \cup V)$, which imply that $U \cup V=d_{X}(U \cup V)$.

(iii): Let $U \in D(X)$ such that (1) $U=d_{X}(U)$. Then, it is verified that $\sim_{g} U \subseteq G_{R}\left(\sim_{g} U\right)$. Indeed, let $x \in \sim_{g} U$ and (2) $y \in R(x)$. Then, $x \in X \backslash g(U)$ and hence (3) $x \notin g(U)$. Suppose that $y \in g(U)$, then there is $z \in U$ such that $y=g(z)$, and by (tS11) in Lemma 9, we get that $R^{-1}(y)=R^{-1}(g(z))=g\left(R^{-1}(z)\right)$. Since $z \in U$, from (1) it follows that $R^{-1}(z) \subseteq U$ and so $\left.g\left(R^{-1}(z)\right)\right) \subseteq g(U)$. Thus, $R^{-1}(y) \subseteq g(U)$. From the last statement and (2), we infer that $x \in g(U)$, which contradicts (3). Consequently, $y \in \sim_{g} U$, which allows us to assert that $R(x) \subseteq \sim_{g} U$ and therefore $\sim_{g} U \subseteq G_{R}(\sim U)$. In a similar way, we can prove that $\sim_{g} U \subseteq H_{R^{-1}}\left(\sim_{g} U\right)$. From the two last assertions we conclude that $\sim_{g} U=d_{X}\left(\sim_{g} U\right)$.

(iv): If $W \in D(X)$ and $d_{X}\left(f_{i_{0} j_{0}}^{-1}(W)\right)=f_{i_{0} j_{0}}^{-1}(W)$ for some $\left(i_{0}, j_{0}\right) \in[n] \times$ $[m]$, then from $(\mathrm{d} 7)$ it follows that $f_{i_{0} j_{0}}^{-1}\left(d_{X}(W)\right)=f_{i_{0} j_{0}}^{-1}(W)$. From the last assertion and (LP5) we infer that $f_{i j}^{-1}\left(d_{X}(W)\right)=f_{i j}^{-1}(W)$ for all $(i, j) \in$ $[n] \times[m]$, and so from $(\mathrm{d} 7)$, we get that $d_{X}\left(f_{i j}^{-1}(W)\right)=f_{i j}^{-1}(W)$ for all $(i, j) \in[n] \times[m]$.

Corollary 11. Let $\left(A, \sim,\left\{\sigma_{i j}\right\}_{(i, j) \in[n] \times[m]}, G, H\right)$ be a tense $L M_{n \times m^{-}}$ algebra. Then, for all $a, b, c \in A$, such that $a=d(a), b=d(b)$ and $\varphi_{i_{0} j_{0}}(c)=d\left(\varphi_{i_{0} j_{0}}(c)\right)$ for some $\left(i_{0}, j_{0}\right) \in[n] \times[m]$, the following properties are satisfied: 
(i) $d(a \wedge b)=a \wedge b$,

(ii) $d(a \vee b)=a \vee b$,

(iii) $d(\sim a)=\sim a$,

(iv) $\sigma_{i j}(c)=d\left(\sigma_{i j}(c)\right)$ for all all $(i, j) \in[n] \times[m]$.

Proof: It is a direct consequence of Lemmas 14 and 19.

Lemma 20. Let $(A, G, H)$ be a tense $L M_{n \times m}$-algebra. Then, for all $a \in A$, the following conditions are equivalent:

(i) $a=d(a)$,

(ii) $a=d^{n}(a)$ for all $n \in \omega$.

Proof: It immediately follows from Corollary 9.

Lemma 21. Let $\left(A, \sim,\left\{\sigma_{i j}\right\}_{(i, j) \in[n] \times[m]}, G, H\right)$ be a tense $L M_{n \times m}$-algebra and $C(A):=\{a \in A: d(a)=a\}$. Then, $\left\langle C(A), \vee, \wedge, \sim,\left\{\sigma_{i j}\right\}_{(i, j) \in[n] \times[m]}, 0,1\right\rangle$ is an $L M_{n \times m}$-algebra.

PRoof: From Corollary 11 and property (d1) in Corollary 9, we have that $\langle C(A), \vee, \wedge, \sim, 0,1\rangle$ is a De Morgan algebra. Taking into account that $a=d(a)$ for all $a \in C(A)$, and the property (iv) in Corollary 11 it follows that $\sigma_{i j}(a)=\sigma_{i j}(d(a))=d\left(\sigma_{i j}(a)\right)$ for all $a \in C(A)$ and $(i, j) \in[n] \times[m]$. Therefore, $\sigma_{i j}(a) \in C(A)$ for all $a \in C(A)$ and $(i, j) \in[n] \times[m]$, from which we conclude that $\left\langle C(A), \vee, \wedge, \sim,\left\{\sigma_{i j}\right\}_{(i, j) \in[n] \times[m]}, 0,1\right\rangle$ is an $L M_{n \times m^{-}}$ algebra.

Corollary 12. Let $(A, G, H)$ be a tense $L M_{n \times m}$-algebra. Then, the structure $(B(C(A)), G, H)$ is a tense Boolean algebra, where $B(C(A))$ is the Boolean algebra of all complemented elements of $C(A)$.

Proof: It is a direct consequence of Lemmas 5 and 21 and property (iv) in Corollary 11.

Remark 4. Let us recall that under the Priestley duality, the lattice of all filters of a bounded distributive lattice is dually isomorphic to the lattice of all increasing closed subsets of the dual space. Under that isomorphism, 
any filter $T$ of a bounded distributive lattice $A$ corresponds to the increasing closed set

$$
Y_{T}=\{S \in X(A): T \subseteq S\}=\bigcap\left\{\sigma_{A}(a): a \in T\right\}
$$

and $\Theta_{C}\left(Y_{T}\right)=\Theta(T)$, where $\Theta_{C}$ is defined as in (2.11) and $\Theta(T)$ is the lattice congruence associated with $T$.

Conversely any increasing closed subset $Y$ of $X(A)$ corresponds to the filter

$$
T_{Y}=\left\{a \in A: Y \subseteq \sigma_{A}(a)\right\},
$$

and $\Theta\left(T_{Y}\right)=\Theta_{C}(Y)$, where $\Theta_{C}$ is defined as in (2.11), and $\Theta\left(T_{Y}\right)$ is the lattice congruence associated with $T_{Y}$.

Taking into account these last remarks on Priestley duality, Theorem 5 and Proposition 5, we can say that the congruences on a tense $L M_{n \times m^{-}}$ algebra are the lattice congruences associated with certain filters of this algebra. So our next goal is to determine the conditions that a filter of a tense $L M_{n \times m}$-algebra must fulfill for the associated lattice congruence to be a tense $L M_{n \times m^{-} \text {-congruence. }}$

TheOREM 6. Let $\left(A, \sim,\left\{\sigma_{i j}\right\}_{(i, j) \in[n] \times[m]}, G, H\right)$ be a tense $L M_{n \times m}$-algebra. If $S$ is a filter of $A$, then, the following conditions are equivalent:

(i) $\Theta(S) \in \operatorname{Con}_{t L M_{n \times m}}(A)$,

(ii) $d\left(\sigma_{i j}(a)\right) \in S$ for any $a \in S$ and $(i, j) \in[n] \times[m]$,

(iii) $d^{n}\left(\sigma_{i j}(a)\right) \in S$ for any $a \in S, n \in \omega$ and $(i, j) \in[n] \times[m]$.

Proof: (i) $\Rightarrow$ (ii): Let $S$ be a filter of $A$ such that $\Theta(S) \in \operatorname{Con}_{t L M_{n \times m}}(A)$. Then, from Priestley duality and Theorem 5 it follows that $\Theta(S)=\Theta_{M T}\left(Y_{S}\right)$, where $\Theta(S)$ is the lattice congruence associated with $S$, and $Y_{S}=\{x \in X(A): S \subseteq x\}=\bigcap_{a \in S} \sigma_{A}(a)$ is a closed, modal and tense subset of the tense $L M_{n \times m}$-space $X(A)$, associated with $A$. Since $Y_{S}$ is modal and $\sigma_{A}$ is an $L M_{n \times m}$-isomorphism, then $Y_{S}=f_{i j}^{A^{-1}}\left(Y_{S}\right)=$ $f_{i j}^{A^{-1}}\left(\bigcap_{a \in S} \sigma_{A}(a)\right)=\bigcap_{a \in S} \sigma_{A}\left(\sigma_{i j}(a)\right)$ for any $(i, j) \in[n] \times[m]$. From the last assertion, and taking into account that $Y$ is a tense subset, Lemmas 17 and 9, Corollary 10 and the fact that the function $d_{X(A)}$ : $X(A) \longrightarrow X(A)$ is monotone, we infer that $Y_{S}=d_{X(A)}\left(\bigcap_{a \in S} \sigma_{A}\left(\sigma_{i j}(a)\right)\right) \subseteq$ 
$\bigcap_{a \in S} d_{X(A)}\left(\sigma_{A}\left(\sigma_{i j}(a)\right)\right)=\bigcap_{a \in S} \sigma_{A}\left(d\left(\sigma_{i j}(a)\right)\right) \subseteq \bigcap_{a \in S} \sigma_{A}\left(\sigma_{i j}(a)\right)=Y_{S}$, for any $(i, j) \in[n] \times[m]$. Hence $Y_{S}=\bigcap_{a \in S} \sigma_{A}\left(d\left(\sigma_{i j}(a)\right)\right.$ for any $(i, j) \in$ $[n] \times[m]$, from which we conclude that $d\left(\sigma_{i j}(a)\right) \in S$ for any $a \in S$ and $(i, j) \in[n] \times[m]$. Indeed, assume that $a \in S$, then $a \in x$ for all $x \in Y_{S}$, from which it follows that $x \in \bigcap_{a \in S} \sigma_{A}\left(d\left(\sigma_{i j}(a)\right)\right.$ for any $(i, j) \in[n] \times[m]$, and thus $d\left(\sigma_{i j}(a)\right) \in x$ for all $x \in Y_{S}$ and $(i, j) \in[n] \times[m]$. Therefore, $d\left(\sigma_{i j}(a)\right) \in \bigcap_{x \in Y_{S}} x$ for any $(i, j) \in[n] \times[m]$, and taking into account that $S=\bigcap_{x \in Y_{S}} x$, we obtain that $d\left(\sigma_{i j}(a)\right) \in S$ for any $(i, j) \in[n] \times[m]$.

(ii) $\Rightarrow$ (i): From Priestley duality and (4.3), we have that $\bigcap_{a \in S} \sigma_{A}(a)=$ $Y_{S}=\{x \in X(A): S \subseteq x\}$ is an increasing and closed subset of $X(A)$ and $\Theta(S)=\Theta\left(Y_{S}\right)$. By Theorem 5, it remains to show that $Y_{S}$ is a modal and tense subset of $X(A)$. From the hypothesis (ii), it follows that for all $a \in S$, $(i, j) \in[n] \times[m]$ and $x \in Y_{S}, d\left(\sigma_{i j}(a)\right) \in x$. Therefore, from this last fact and Corollary 11, it results that $\sigma_{i j}(d(a)) \in x$ for all $(i, j) \in[n] \times[m]$ and all $x \in Y_{S}$, and hence $(1) Y_{S} \subseteq \bigcap_{a \in S} \sigma_{A}\left(\sigma_{i j}(d(a))\right)$ for all $(i, j) \in[n] \times[m]$. Consequently, by Corollary $9, Y_{S} \subseteq \bigcap_{a \in S} \sigma_{A}\left(\sigma_{i j}(a)\right)$ for all $(i, j) \in[n] \times[m]$, and from this assertion it follows that $Y_{S} \subseteq \bigcap_{a \in S} \sigma_{A}\left(\varphi_{1}(a)\right) \subseteq \bigcap_{a \in S} \sigma_{A}(a)=$ $Y_{s}$. Since $\sigma_{A}$ is an $L M_{n \times m}$-isomorphism, then we get that (2) $Y_{s}=$ $\bigcap_{a \in S} \sigma_{A}\left(\sigma_{11}(a)\right)=\bigcap_{a \in S} f_{11}^{A^{-1}}\left(\sigma_{A}(a)\right)=f_{11}^{A^{-1}}\left(\bigcap_{a \in S} \sigma_{A}(a)\right)=f_{11}^{A^{-1}}\left(Y_{S}\right)$. Therefore from the last statement and (LP6) we conclude that $Y_{S}=f_{i j}^{A}\left(Y_{S}\right)$ for all $(i, j) \in[n] \times[m]$ and so $Y_{S}$ is modal. In addition, from (1), (2) and Corollary 9 we infer that $Y_{S} \subseteq \bigcap_{a \in S} \sigma_{A}\left(d\left(\sigma_{11}(a)\right) \subseteq \bigcap_{a \in S} \sigma_{A}\left(\sigma_{11}(a)\right)=Y_{S}\right.$ and hence, $Y_{S}=\bigcap_{a \in S} \sigma_{A}\left(d\left(\sigma_{11}(a)\right)\right.$. Then, taking into account Corollary 10 and that $\bigcap_{a \in S} d_{X(A)}\left(\sigma_{A}\left(\sigma_{11}(a)\right)\right)=d_{X(A)}\left(\bigcap_{a \in S} \sigma_{A}\left(\sigma_{11}(a)\right)\right)$, we obtain that $Y_{S}=d_{X(A)}\left(Y_{S}\right)$, and thus, from Lemma 17 and the fact that $Y_{S}$ is modal, we infer that $Y_{S}$ is a tense subset of $X(A)$. Finally, since $Y_{S}$ is a closed, modal and tense subset of $X(A)$ and $\Theta(S)=\Theta_{M T}\left(Y_{S}\right)$, we conclude, from Theorem 5, that $\Theta(S) \in \operatorname{Con}_{t L M_{n \times m}}(A)$.

(ii) $\Leftrightarrow$ (iii): It is trivial. 
Theorem 6 leads us to introduce the following definition:

Definition 18. Let $(A, G, H)$ be a tense $L M_{n \times m}$-algebra. A filter $S$ of $A$ is a tense filter iff

(tf) $d(a) \in S$ for all $a \in S$ or equivalently $d^{n}(a) \in S$ for all $a \in S$ and $n \in \omega$.

Now, we remember the notion of Stone filter of an $L M_{n \times m}$-algebra. Definition 19. Let $\left(A, \sim,\left\{\sigma_{i j}\right\}_{(i, j) \in[n] \times[m]}\right)$ be an $L M_{n \times m}$-algebra. A filter $S$ of $A$ is a Stone filter iff

(sf) $\sigma_{i j}(a) \in S$ for all $a \in S$ and $(i, j) \in[n] \times[m]$, or equivalently $\sigma_{11}(a) \in S$ for all $a \in S$.

LEMMA 22. Let $\left(A, \sim,\left\{\sigma_{i j}\right\}_{(i, j) \in[n] \times[m]}, G, H\right)$ be a tense $L M_{n \times m}$-algebra. If $S$ is a Stone filter of $A$, then the following conditions are equivalent:

(i) $S$ is a tense filter of $A$,

(ii) $d^{n}\left(\sigma_{i j}(a)\right) \in S$ for all $a \in S, n \in \omega$ and $(i, j) \in[n] \times[m]$.

\section{ProOF:}

(i) $\Rightarrow$ (ii): Let $S$ be a Stone filter of $A, a \in S, n \in \omega$ and $(i, j) \in[n] \times[m]$. Since $S$ is an Stone filter of $A$, we have that $\sigma_{i j}(a) \in S$. From this last assertion and the fact that $S$ is a tense filter we conclude that $d^{n}\left(\sigma_{i j}(a)\right) \in$ $S$.

(ii) $\Rightarrow$ (i): Let $a \in S$. Then, from the hypothesis (ii) we obtain that $d^{n}\left(\sigma_{11}(a)\right) \in S$. From the last assertion, properties (C13) and (d5) and the fact that $S$ is a filter of $A$ we infer that $d^{n}(a) \in S$ for all $n \in \omega$, and therefore $S$ is a tense filter of $A$.

We will denote by $F_{T S}(A)$ the set of all tense Stone filters of a tense $L M_{n \times m}$-algebra $(A, G, H)$.

Proposition 9. Let $\left(A, \sim,\left\{\sigma_{i j}\right\}_{(i, j) \in[n] \times[m]}, G, H\right)$ be a tense $L M_{n \times m^{-}}$ algebra. Then, the following conditions are equivalent for all $\theta \subseteq A \times A$ :

(i) $\theta \in \operatorname{Con}_{t L M_{n \times m}}(A)$,

(ii) there is $S \in F_{T S}(A)$ such that $\theta=\Theta(S)$, where $\Theta(S)$ is the lattice congruence associated with the filter $S$.

Proof:

(i) $\Rightarrow$ (ii): From (i) and Theorem 5, it follows that there exists $Y \in$ $C_{M T}(X(A))$ such that $(1) \Theta_{M T}(Y)=\theta$. Then, from Remark 4, we infer that $T_{Y}=\left\{a \in A: Y \subseteq \sigma_{A}(a)\right\}$ is a filter on $A$ and $(2) \Theta\left(T_{Y}\right)=$ 
$\Theta(Y)=\Theta_{M T}(Y)$. Therefore $\Theta\left(T_{Y}\right) \in \operatorname{Con}_{t L M_{n \times m}}(A)$, and so from Theorem 6, we obtain that $Y \in F_{T S}(A)$. This last assertion, (1) and (2) enable us to conclude the proof.

(ii) $\Rightarrow$ (i): It immediatly follows from Theorem 6 .

Corollary 13. Let $(A, G, H)$ be a tense $L M_{n \times m}$-algebra. Then,

(i) $(A, G, H)$ is a simple tense $L M_{n \times m}$-algebra if and only if $F_{T S}(A)=$ $\{A,\{1\}\}$.

(ii) $(A, G, H)$ is a subdirectly irreducible tense $L M_{n \times m}$-algebra if and only if there is $T \in F_{T S}(A), T \neq\{1\}$ such that $T \subseteq S$ for all $S \in F_{T S}(A), S \neq\{1\}$.

Proof: It is a direct consequence of Corollaries 7 and 8, Remark 4 and Proposition 9.

Finally, we will describe the simple and subdirectly irreducible tense $L M_{n \times m}$-algebras.

In the proof of the following proposition we will use the finite intersection property of compact spaces, which establishes that if $X$ is a compact topological space, then for each family $\left\{M_{i}\right\}_{i \in I}$ of closed subsets of $X$ satisfying $\bigcap_{i \in I} M_{i}=\emptyset$, there is a finite subfamily $\left\{M_{i_{1}}, \ldots, M_{i_{n}}\right\}$ such that $\bigcap_{j=1}^{n} M_{i_{j}}=\emptyset$.

Proposition 10. Let $(A, G, H)$ be a tense $L M_{n \times m}$-algebra and $\left(X(A), g_{A}\right.$, $\left.\left\{f_{i j}^{A}\right\}_{(i, j) \in[n] \times[m]}, R^{A}\right)$ be the tense $L M_{n \times m}$-space associated with $A$. Then, the following conditions are equivalent:

(i) $(A, G, H)$ is a simple tense $L M_{n \times m}$-algebra,

(ii) for every $U \in D(X(A)) \backslash\{X(A)\}$ and for every $(i, j) \in[n] \times[m]$ such that $f_{i j}^{A^{-1}}(U) \neq X(A), \bigcap_{n \in \omega} d_{X(A)}^{n}\left(f_{i j}^{A^{-1}}(U)\right)=\emptyset$

(iii) for every $U \in D(X(A)) \backslash\{X(A)\}$ and for every $(i, j) \in[n] \times[m]$ such that $f_{i j}^{A^{-1}}(U) \neq X(A), d_{X(A)}^{n_{i j}^{U}}\left(f_{i j}^{A^{-1}}(U)\right)=\emptyset$ for some $n_{i j}^{U} \in \omega$,

(iv) for every $U \in B(D(X(A))) \backslash\{X(A)\}$, there is $n_{U} \in \omega$ such that $d_{X(A)}^{n_{U}}(U)=\emptyset$

(v) $F_{T S}(D(X(A)))=\{D(X(A)),\{X(A)\}\}$. 


\section{ProOF:}

(i) $\Rightarrow$ (ii): Let $U \in D(X(A)) \backslash\{X(A)\}$. Now, let $(i, j) \in[n] \times[m]$ such that $f_{i j}^{A^{-1}}(U) \neq X(A)$, then from (d5) in Proposition 8 we have that $d_{X(A)}^{n}\left(f_{i j}^{A^{-1}}(U)\right) \neq X(A)$. From this last assertion and (d9) in Proposition 8, we obtain that $\bigcap_{n \in \omega} d_{X(A)}^{n}\left(f_{i j}^{A^{-1}}(U)\right) \in C_{M T}(X(A)) \backslash\{X(A)\}$. From this last assertion, the hypothesis (i) and Corollary 7 , we conclude that $\bigcap_{n \in \omega} d_{X(A)}^{n}\left(f_{i j}^{A^{-1}}(U)\right)=\emptyset$.

(ii) $\Rightarrow$ (iii): Let $U \in D(X(A)) \backslash\{X(A)\}$ and $(i, j) \in[n] \times[m]$ such that $f_{i j}^{A^{-1}}(U) \neq X(A)$. Then, from the hypothesis (ii), we have that

$$
\text { (1) } \bigcap_{n \in \omega} d_{X(A)}^{n}\left(f_{i j}^{A^{-1}}(U)\right)=\emptyset \text {. }
$$

Besides, for all $n \in \omega, d_{X(A)}^{n}\left(f_{i j}^{A^{-1}}(U)\right)$ is a closed subset of $X(A)$ and $d_{X(A)}^{n}\left(f_{i j}^{A^{-1}}(U)\right)=\bigcap_{k=1}^{n} d_{X(A)}^{k}\left(f_{i j}^{A^{-1}}(U)\right)$. Then, from (1), the last statement, the fact that $X(A)$ is compact and the finite intersection property of compact spaces, we conclude that there is $n_{i j}^{U} \in \omega$ such that $d_{X(A)}^{n_{i j}^{U}}\left(f_{i j}^{A^{-1}}(U)\right)=\emptyset$.

(iii) $\Rightarrow$ (iv): From Lemma 5, we have that $U \in B(D(X(A))$ ) if and only if $U=f_{i j}^{A^{-1}}(U)$ for all $(i, j) \in[n] \times[m]$, and so from property (LP10) of $L M_{n \times m}$-spaces, we infer that $U \in B(D(X(A))) \backslash\{X(A)\}$ iff $f_{i j}^{A^{-1}}(U) \neq$ $X(A)$ for all $(i, j) \in[n] \times[m]$. Therefore, from the previous assertion and the hypothesis (iii), we obtain that for each $U \in B(D(X(A)))$ and each $(i, j) \in[n] \times[m]$, there is $n_{i j}^{U} \in \omega$ such that $d_{X(A)}^{n_{i j}^{U}}(U)=\emptyset$. Since, from (1) it follows that for all $(i, j),(r, s) \in[n] \times[m], n_{i j}^{U}=n_{r s}^{U}=n^{U}$, then the proof is complete.

(iv) $\Rightarrow$ (v): Assume that $S \in F_{T S}(D(X(A))), S \neq\{X(A)\}$. Then there is (1) $U \in S, U \neq X(A)$ and so from property (LP10) of $L M_{n \times m}$-spaces, we infer that there is $(i, j) \in[n] \times[m]$ such that $f_{i j}^{A^{-1}}(U) \neq X(A)$. Considering (2) $V=f_{i j}^{A^{-1}}(U)$, then from Lemma 5, we obtain that $V \in B(D(X(A)))$, $V \neq X(A)$. Hence, from the hypothesis (iv), we can assert that there is $n_{V} \in \omega$ such that $d_{X(A)}^{n_{V}}(V)=\emptyset$. From (1), (2), the preceding assertion and Definitions 18 and 19, we deduce that $\emptyset \in S$, which implies that $S=D(X(A))$. 
$(\mathrm{v}) \Rightarrow(\mathrm{i})$ : It immediately follows from Corollary 13 and the fact that $(A, G, H)$ is isomorphic to the tense $L M_{n \times m^{-a l g e b r a}}$ $\left(D(X(A)), G_{R^{A}}, H_{R^{A-1}}\right)$.

Corollary 14. Let $\left(A, \sim,\left\{\sigma_{i j}\right\}_{(i, j) \in[n] \times[m]}, G, H\right)$ be a tense $L M_{n \times m^{-}}$ algebra. Then, the following conditions are equivalent:

(i) $\left(A, \sim,\left\{\sigma_{i j}\right\}_{(i, j) \in[n] \times[m]}, G, H\right)$ is a simple tense $L M_{n \times m}$-algebra,

(ii) for every $a \in A \backslash\{1\}$ and for every $(i, j) \in[n] \times[m]$ such that $\sigma_{i j}(a) \neq 1, d^{n_{i j}^{a}}\left(\sigma_{i j}(a)\right)=0$ for some $n_{i j}^{a} \in \omega$,

(iii) for each $a \in B(A) \backslash\{1\}$, there is $n_{a} \in \omega$ such that $d^{n_{a}}(a)=0$,

(iv) $F_{T S}(A)=\{A,\{1\}\}$.

Proof: It is a direct consequence of Proposition 10 and the fact that $\sigma_{A}: A \longrightarrow D(X(A))$ is a tense $L M_{n \times m}$-isomorphism.

Corollary 15. If $\left(A, \sim,\left\{\sigma_{i j}\right\}_{(i, j) \in[n] \times[m]}, G, H\right)$ is a simple tense $L M_{n \times m^{-}}$ algebra, then $B(C(A))=\{0,1\}$ and therefore $\left(C(A), \sim,\left\{\sigma_{i j}\right\}_{(i, j) \in[n] \times[m]}\right)$ is a simple $L M_{n \times m}$-algebra.

Proof: From Lemmas 5 and 20, property (iv) in Corollary 11 and property (ii) in Corollary 14 it follows that $B(C(A))=\{0,1\}$. From this last assertion, (LM8) and Lemma 21 the proof is complete.

Next, we will recall two concepts which will play a fundamental role in this paper. Let $Y$ be a topological space and $y_{0} \in Y$. A net in a space $Y$ is a map $\varphi: D \longrightarrow Y$ of some directed set $(D, \prec)$ (i.e. $D \neq \emptyset$ and $\prec$ is a preorder on $D$ and for all $d_{1}, d_{2} \in D$ there is $d_{3} \in D$ such that $d_{1} \prec d_{3}$ and $d_{2} \prec d_{3}$ ). Besides, we say that $\varphi$ converges to $y_{0}$ (written $\varphi \rightarrow y_{0}$ ) if for all neighborhoods $U\left(y_{0}\right)$ of $y_{0}$ there is $d_{0} \in D$ such that for all $d \in D, d_{0} \prec d$, $\varphi(d) \in U\left(y_{0}\right)$. We also say that $\varphi$ accumulates at $y_{0}$ (written $\varphi \succ y_{0}$ ) if for all neighborhoods $U\left(y_{0}\right)$ of $y_{0}$ and for all $d \in D$, there is $d_{c} \in D$ such that $d \prec d_{c}$ and $\varphi\left(d_{c}\right) \in U\left(y_{0}\right)$. If $\varphi: D \longrightarrow Y$ is a net and $y_{d}=\varphi(d)$ for all $d \in D$, then the net $\varphi$ it will be denoted by $\left(y_{d}\right)_{d \in D}$. If $\varphi \rightarrow y_{0}$, it will be denoted by $\left(y_{d}\right) \underset{d \in D}{\longrightarrow} y_{0}$. If $\varphi \succ y_{0}$, it will be denoted $\left(y_{d}\right)_{d \in D} \succ y_{0}$.

Proposition 11. Let $(A, G, H)$ be a tense $L M_{n \times m}$-algebra and $\left(X(A), g_{A}\right.$, $\left.\left\{f_{i j}^{A}\right\}_{(i, j) \in[n] \times[m]}, R^{A}\right)$ be the tense $L M_{n \times m}$-space associated with $A$. Then, the following conditions are equivalent: 
(i) $(A, G, H)$ is a subdirectly irreducible tense $L M_{n \times m}$-algebra,

(ii) there is $V \in B(D(X(A))), V \neq X(A)$, such that for each $U \in$ $D(X(A)), U \neq X(A)$ and for each $(i, j) \in[n] \times[m]$ such that $f_{i j}^{A^{-1}}(U) \neq X(A), \bigcap_{n \in \omega} d_{X(A)}^{n}\left(f_{i j}^{A^{-1}}(U)\right) \subseteq V$,

(iii) there is $V \in B(D(X(A))), V \neq X(A)$, such that for each $U \in$ $D(X(A)), U \neq X(A)$ and for each $(i, j) \in[n] \times[m]$ such that $f_{i j}^{A^{-1}}(U) \neq X(A), d_{X(A)}^{n_{i}^{U}}\left(f_{i j}^{A^{-1}}(U)\right) \subseteq V$ for some $n_{i j}^{U} \in \omega$,

(iv) there is $V \in B(D(X(A))), V \neq X(A)$, such that for all $U \in$ $B(D(X(A))), U \neq X(A), d_{X(A)}^{n^{U}}(U) \subseteq V$, for some $n^{U} \in \omega$,

(v) there is $T \in F_{T S}(D(X(A)), T \neq\{X(A)\}$, such that $T \subseteq S$ for all $S \in F_{T S}(D(X(A))), S \neq\{X(A)\}$.

Proof:

(i) $\Rightarrow$ (ii): From (i) and Corollary 8 we infer that there exists $Y \in C_{M T}(X(A)) \backslash\{X(A)\}$ such that (1) $Z \subseteq Y$ for all $Z \in C_{M T}(X(A)) \backslash$ $\{X(A)\}$. Since $Y$ is modal, then by Proposition 5 , there is (2) $x \in$ $\max X(A) \backslash Y$. Taking into account that $Y$ is a closed subset of $X(A)$ and hence it is compact, we can assert that there is $W \in D(X(A))$, such that (3) $Y \subseteq W$ and (4) $x \notin W$. In addition from (2) and (LP15) in Corollary 1, we have that $x=f_{(n-1)(m-1)}^{A}(x)$ and so by (4) we infer that $x \notin$ $f_{(n-1)(m-1)}^{A^{-1}}(W)$. If $V=f_{(n-1)(m-1)}^{A^{-1}}(W)$, then $V \in B(D(X(A))) \backslash\{X(A)\}$. Besides, from (3) and the fact that $Y=f_{(n-1)(m-1)}^{A^{-1}}(Y)$, we get that (5) $Y \subseteq f_{(n-1)(m-1)}^{A^{-1}}(W)=V$. On the other hand, if $U \in D(X(A)) \backslash\{X(A)\}$, then from Lemma 14 and property (LP10) of $L M_{n \times m}$-spaces, we infer that there is at least $\left(i_{0}, j_{0}\right) \in[n] \times[m]$ such that $f_{i_{0} j_{0}}^{A^{-1}}(U) \neq X(A)$. Now, let $(i, j) \in[n] \times[m]$ such that $f_{i j}^{A^{-1}}(U) \neq X(A)$, then from Proposition 8 we obtain that $\bigcap_{n \in \omega} d_{X(A)}^{n}\left(f_{i j}^{A^{-1}}(U)\right) \in C_{M T}(X(A)) \backslash\{X(A)\}$, from which we conclude, by the assertions (1) and (5), that $\bigcap_{n \in \omega} d_{X(A)}^{n}\left(f_{i j}^{A^{-1}}(U)\right) \subseteq V$.

(ii) $\Rightarrow$ (iii): From the hypothesis (ii), we have that there is $V \in$ $B(D(X(A))) \backslash\{X(A)\}$, such that $(1) \bigcap_{n \in \omega} d_{X(A)}^{n}\left(f_{i j}^{A^{-1}}(U)\right) \subseteq V$ for each $U \in D(X(A)) \backslash\{X(A)\}$ and each $i \in[n] \times[m]$ such that $f_{i j}^{A^{-1}}(U) \neq X(A)$. Suppose that there is $U \in D(X(A)) \backslash\{X(A)\}$ and there is $i_{0} \in[n] \times[m]$, 
which satisfy (1) and $d_{X(A)}^{n}\left(f_{i_{0} j_{0}}^{A}{ }^{-1}(U)\right) \nsubseteq V$ for all $n \in \omega$. Then for each $n \in \omega$, there exists $(2) x_{n} \in d_{X(A)}^{n}\left(f_{i_{0} j_{0}}^{A^{-1}}(U)\right)$ and $x_{n} \notin V$. Hence $\left(x_{n}\right)_{n \in \omega}$ is a sequence in $X(A) \backslash V$ and since $X(A) \backslash V$ is compact, we can assert that there exists (3) $x \in X(A) \backslash V$ such that $\left(x_{n}\right)_{n \in \omega}$ accumulates at $x$. In addition, by (1) and (3), we have that $x \notin \bigcap_{n \in \omega} d_{X(A)}^{n}\left(f_{i_{0} j_{0}}^{A}{ }^{-1}(U)\right)$, and thus $x \in X(A) \backslash d_{X(A)}^{n_{0}}\left(f_{i_{0} j_{0}}^{A^{-1}}(U)\right)$ for some $n_{0} \in \omega$. Since $x$ is an accumulation point of $\left(x_{n}\right)_{n \in \omega}$, then the preceding assertion and the fact that $X(A) \backslash d_{X(A)}^{n_{0}}\left(f_{i_{0} j_{0}}^{A^{-1}}(U)\right)$ is an open subset of $X(A)$ allows us to infer that for all $n \in \omega$ there is $m_{n} \in \omega$ such that $n \leq m_{n}$ and $x_{m_{n}} \in X(A) \backslash$ $d_{X(A)}^{n_{0}}\left(f_{i_{0} j_{0}}^{A^{-1}}(U)\right)$. Thus $x_{m_{n_{0}}} \in X(A) \backslash d_{X(A)}^{n_{0}}\left(f_{i_{0} j_{0}}^{A^{-1}}(U)\right)$ and $n_{0} \leq m_{n_{0}}$. As a consequence of Proposition 8 we have that $X(A) \backslash d_{X(A)}^{n_{0}}\left(f_{i_{0} j_{0}}^{A^{-1}}(U)\right) \subseteq$ $X(A) \backslash d_{X(A)}^{m_{n_{0}}}\left(f_{i_{0} j_{0}}^{A^{-1}}(U)\right)$ and so $\left.x_{m_{n_{0}}} \notin d_{X(A)}^{m_{n_{0}}}\left(f_{i_{0} j_{0}}^{A^{-1}}(U)\right)\right)$, which contradicts (2). Therefore, for every $U \in D(X(A)) \backslash\{X(A)\}$ and $(i, j) \in[n] \times[m]$ such that $f_{i j}^{A^{-1}}(U) \neq X(A), d_{X(A)}^{n_{i}^{U}}\left(f_{i j}^{A^{-1}}(U)\right) \subseteq V$ for some $n_{i}^{U} \in \omega$.

(iii) $\Rightarrow$ (iv): From Lemma 5 and the property (LP10) of $L M_{n \times m}$-spaces, we infer that for all $U \in B(D(X(A))), U \neq X(A)$ if and only if $f_{i j}^{A^{-1}}(U) \neq$ $X(A)$ for all $(i, j) \in[n] \times[m]$. Therefore, from the last statement and the hypothesis (iii), we obtain that for each $U \in B(D(X(A))), U \neq X(A)$ and each $(i, j) \in[n] \times[m]$, there is $n_{i j}^{U} \in \omega$ such that $d_{X(A)}^{n_{i j}^{U}}(U) \subseteq V$. Then, considering $n_{U}=\max \left\{n_{i j}^{U}:(i, j) \in[n] \times[m]\right\}$, from (d2) in Proposition 8 we conclude that $d_{X(A)}^{n_{U}}(U) \subseteq V$.

(iv) $\Rightarrow\left(\right.$ v): Let $S \in F_{T S}(D(X(A))), S \neq\{X(A)\}$. Then there exists (1) $U \in S \backslash\{X(A)\}$ and so from property (LP10) we infer that there is $(i, j) \in[n] \times[m]$ such that $f_{i j}^{A^{-1}}(U) \neq X(A)$. Let $(2) W=f_{i j}^{A^{-1}}(U)$. Then, from Lemma 5 we have that $W \in B(D(X(A))), W \neq X(A)$ and thus by the hypothesis (iv), we can assert that there is $n_{W} \in \omega$ such that (3) $d_{X(A)}^{n_{W}}(W) \subseteq V$. Besides, from the assertions (1) and (2) and Lemma 22 , we obtain that $d_{X(A)}^{n_{W}}(W) \in S$. From the last statement, (3) and the fact that $S$ is a filter of $D(X(A))$, we get that $V \in S$, and so $V \in \bigcap_{S \in \Omega} S$, where $\Omega=\left\{S \in F_{T S}(D(X(A))): S \neq\{X(A)\}\right\}$. Therefore, considering $T=\bigcap_{S \in \Omega} S$ and taking into account that $V \neq X(A)$, we conclude that $T \in \Omega$ and $T \subseteq S$, for all $S \in \Omega$. 
(v) $\Rightarrow$ (i): It follows from the fact that $(A, G, H)$ and

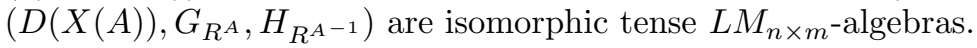

Corollary 16. Let $\left(A, \sim,\left\{\sigma_{i j}\right\}_{(i, j) \in[n] \times[m]}, G, H\right)$ be a tense $L M_{n \times m^{-}}$ algebra. Then, the following conditions are equivalent:

(i) $\left(A, \sim,\left\{\sigma_{i j}\right\}_{(i, j) \in[n] \times[m]}, G, H\right)$ is a subdirectly irreducible tense $L M_{n \times m}$-algebra,

(ii) there is $b \in B(A) \backslash\{1\}$ such that for every $a \in A \backslash\{1\}$ and for every $(i, j) \in[n] \times[m]$ such that $\sigma_{i j}(a) \neq 1, d^{n_{i j}^{a}}\left(\sigma_{i j}(a)\right) \leq b$ for some $n_{i j}^{a} \in \omega$,

(iii) there is $b \in B(A) \backslash\{1\}$ such that for every $a \in B(A) \backslash\{1\}$, there is $n_{a} \in \omega$ such that $d^{n_{a}}(a) \leq b$,

(iv) there is $T \in F_{T S}(A), T \neq\{1\}$ such that $T \subseteq S$ for all $S \in F_{T S}(A)$, $S \neq\{1\}$.

Proof: It is a direct consequence of Proposition 11 and the fact that $\sigma_{A}: A \longrightarrow D(X(A))$ is a tense $L M_{n \times m}$-isomorphism.

Corollary 17. Let $\left(A, \sim,\left\{\sigma_{i j}\right\}_{(i, j) \in[n] \times[m]}, G, H\right)$ be a subdirectly irreducible tense $L M_{n \times m}$-algebra such that for every $a \in B(A) \backslash\{1\}, d^{n}(a)=$ $d^{n_{a}}(a)$ for some $n_{a} \in \omega$ and for all $n \in \omega, n_{a} \leq n$. Then, $(C(A) \sim$, $\left.\left\{\sigma_{i j}\right\}_{(i, j) \in[n] \times[m]}\right)$ is a simple $L M_{n \times m}$-algebra.

Proof: From Corollary 16, we can assert that there exists $b \in B(A) \backslash\{1\}$ such that (1) for every $a \in B(A) \backslash\{1\}, \quad d^{n_{a}}(a) \leq b$ for some $n_{a} \in \omega$. Also, from hypothesis we have that there is $n_{b} \in \omega$ such that $d^{n}(b)=d^{n_{b}}(b)$ for all $n \in \omega, n_{b} \leq n$. Considering $u=d^{n_{b}}(b)$, then from the last assertion, properties (d5) and (d7) in Corollary 9 and the fact that $b \in B(A) \backslash\{1\}$, we obtain that $u \in B(C(A)), u \neq 1$. In addition, let $c \in B(C(A)), c \neq 1$, then by Lemma $20, c=d^{n}(c)$ for all $n \in \omega$, and thus from (1) we get that $c=d^{n_{c}}(c) \leq b$. Then from property (d4) in Corollary 9 , we infer that $c=d^{n_{b}}(c) \leq d^{n_{b}}(b)=u$. Consequently, from Corollary $12, B(C(A))$ is a totally ordered Boolean algebra and so $B(C(A))=\{0,1\}$. Therefore, from (LM8) and Lemma 21, we conclude that $\left(C(A) \sim,\left\{\sigma_{i j}\right\}_{(i, j) \in[n] \times[m]}\right)$ is a simple $L M_{n \times m}$-algebra. 


\section{Conclusion and future research}

Priestley spaces arise more naturally in relation with logics, as Priestley spaces incorporate the now widely used Kripke semantics in them. As a result, Priestley's duality became rather popular among logicians, and most dualities for distributive lattices with operators have been performed in terms of Priestley spaces. In particular, in this paper we have determined a topological duality for tense $n \times m$-valued Lukasiewicz-Moisil algebras, extending the one obtained for $n \times m$-valued Lukasiewicz-Moisil algebras in [27]. By means of the above duality we have characterized simple and subdirectly irreducible tense $n \times m$-valued Łukasiewicz-Moisil algebras. We expect that our method can be easily applied to modal operators or monadic operators on $n \times m$-valued Łukasiewicz-Moisil algebras (see, [25], [27]).

\section{References}

[1] V. Boicescu, A. Filipoiu, G. Georgescu and S. Rudeanu, Eukasiewicz-Moisil Algebras, Annals of Discrete Mathematics, Vol. 49 (1991), NorthHolland.

[2] M. Botur, I. Chajda, R. Halaš and M. Kolařík, Tense operators on Basic Algebras, International Journal of Theoretical Physics, Vol. 50, No. 12 (2011), pp. 3737-3749.

[3] M. Botur, J. Paseka, On tense MV-algebras, Fuzzy Sets and Systems, Vol. 259 (2015), pp. 111-125.

[4] J. Burges, Basic tense logic, [in:] D. M. Gabbay, F. Günter (eds.), Handbook of Philosophical Logic, Vol. II, Reidel, Dordrecht (1984), pp. 89139.

[5] I. Chajda, Algebraic axiomatization of tense intuitionistic logic, Central European Journal of Mathematics, Vol. 9, No. 5 (2011), pp. 1185-1191.

[6] I. Chajda and J. Paseka, Dynamic effect algebras and their representations, Soft Computing, Vol. 16, No. 10 (2012), pp. 1733-1741.

[7] I. Chajda and M. Kolařík, Dynamic Effect Algebras, Mathematica Slovaca, Vol. 62, No. 3 (2012), pp. 379-388.

[8] C. Chiriţă, Tense $\theta$-valued Moisil propositional logic, International Journal of Computers Communications and Control, Vol. 5 (2010), pp. 642-653. 
[9] C. Chiriţă, Tense $\theta$-valued Eukasiewicz-Moisil algebras, Journal of Multiple-Valued Logic and Soft Computing, Vol. 17, No. 1 (2011), pp. $1-24$.

[10] R. Cignoli, Moisil Algebras, Notas de Lógica Matemática, Vol. 27 (1970), Instituto de Matemática, Universidad Nacional del Sur, Bahía Blanca.

[11] W. Cornish and P. Fowler, Coproducts of De Morgan algebras, Bulletin of the Australian Mathematical Society, Vol. 16 (1977), pp. 1-13.

[12] D. Diaconescu and G. Georgescu, Tense operators on $M V$-algebras and Eukasiewicz-Moisil algebras, Fundamenta Informaticae, Vol. 81, No. 4 (2007), pp. 379-408.

[13] A. V. Figallo, G. Pelaitay, $n \times m$-valued Eukasiewicz-Moisil algebras with two modal operators, South American Journal of Logic, Vol. 1, No. 1 (2015), pp. 267-281.

[14] A. V. Figallo, I. Pascual, G. Pelaitay, A topological duality for tense $L_{n^{-}}$ algebras and applications, Logic Journal of the IGPL, Vol. 26, No. 4 (2018), pp. 339-380.

[15] A. V. Figallo and G. Pelaitay, Note on tense SHn-algebras, Analele Universitatii din Craiova. Seria Matematica-Informatica, Vol. 38, No. 4 (2011), pp. 24-32.

[16] A. V. Figallo and G. Pelaitay, Tense operators on De Morgan algebras, Logic Journal of the IGPL, Vol. 22, No. 2, (2014), pp. 255-267.

[17] A. V. Figallo and G. Pelaitay, A representation theorem for tense $n \times m$ valued Eukasiewicz-Moisil algebras, Mathematica Bohemica, Vol. 140, No. 3 (2015), pp. 345-360.

[18] A. V. Figallo and G. Pelaitay, Discrete duality for tense Eukasiewicz-Moisil algebras, Fundamenta Informaticae, Vol. 136, No. 4 (2015), pp. 317-329.

[19] T. Kowalski, Varieties of tense algebras, Reports on Mathematical Logic, Vol. 32 (1998), pp. 53-95.

[20] Gr. C. Moisil, Recherches sur les logiques non-chrysippiennes, Annales Scientifiques de l'Université de Jassy, Vol. 26 (1940), pp. 431-466.

[21] J. Paseka, Operators on $M V$-algebras and their representations, Fuzzy Sets and Systems, Vol. 232 (2013), pp. 62-73.

[22] H. A. Priestley, Representation of distributive lattices by means of ordered Stone spaces, Bulletin of the London Mathematical Society, Vol. 2 (1970), pp. 186-190.

[23] A. V. Figallo and C. Sanza, Álgebras de Eukasiewicz $n \times m$-valuadas con negación, Noticiero de la Unión Matemática Argentina, Vol. 93 (2000). 
[24] A. V. Figallo and C. Sanza, The $N S_{n \times m}$-propositional calculus, Bulletin of the Section of Logic, Vol. 35, No. 2 (2008), pp. 67-79.

[25] A. V. Figallo and C. Sanza, Monadic $n \times m$-valued Eukasiewicz-Mosil algebras, Mathematica Bohemica, Vol. 137, No. 4 (2012), pp. 425-447.

[26] A. V. Figallo and G. Pelaitay, A representation theorem for tense $n \times m$ valued Eukasiewicz-Moisil algebras, Mathematica Bohemica, Vol. 140, No. 3 (2015), pp. 345-360.

[27] A. V. Figallo, I. Pascual, G. Pelaitay, A new topological duality for $n \times m$ valued Eukasiewicz-Moisil algebras, Asian-European Journal of Mathematics (2019).

[28] C. Gallardo, C. Sanza and A. Ziliani, F-multipliers and the localization of $L M_{n \times m}$-algebras, Analele Stiintifice ale Universitatii Ovidius Constanta, Vol. 21, No. 1 (2013), pp. 285-304.

[29] Gr. C. Moisil, Essais sur les logiques non Chrysippiennes, Ed. Academiei, Bucarest, 1972.

[30] H. Priestley, Representation of distributive lattices by means of ordered Stone spaces, Bulletin of the London Mathematical Society, Vol. 2 (1970), pp. 186-190.

[31] H. Priestley, Ordered topological spaces and the representation of distributive lattices, Proceedings of the London Mathematical Society, Vol. 3 (1972), pp. 507-530.

[32] H. Priestley, Ordered sets duality for distributive lattices, Annals of Discrete Mathematics, Vol. 23 (1984), pp. 39-60.

[33] C. Sanza, Notes on $n \times m$-valued Eukasiewicz algebras with negation, Logic Journal of the IGPL, Vol. 6, No. 12 (2004), pp. 499-507.

[34] C. Sanza, $n \times m$-valued Eukasiewicz algebras with negation, Reports on Mathematical Logic, Vol. 40 (2006), pp. 83-106.

[35] C. Sanza, On $n \times m$-valued Lukasiewicz-Moisil algebras, Central European Journal of Mathematics, Vol. 6, No. 3 (2008), pp. 372-383.

[36] W. Suchoń, Matrix Lukasiewicz Algebras, Reports on Mathematical Logic, Vol. 4 (1975), pp. 91-104.

Universidad Nacional de San Juan Instituto de Ciencias Básicas 5400 San Juan, Argentina e-mail: avfigallo@gmail.com ipascual756@hotmail.com gpelaitay@gmail.com 